\title{
Efficiency and Stability under Substitutable Priorities with Ties
}

\author{
Aytek Erdil \\ University of Cambridge, Faculty of Economics, UK \\ Taro Kumano*广 \\ Yokohama National University, Department of Economics, Japan
}

September 25, 2019

\begin{abstract}
Many assignment mechanisms appeal to a priority structure to determine how over-subscribed indivisible goods are assigned to unit-demand individuals. We study substitutable priorities with ties which not only nest important classes of priorities and preferences studied in the literature, but also allow us to formalize plausible priority structures not captured in previous literature. Efficiency is typically in conflict with respecting priorities (i.e., stability), and therefore the natural welfare objective is constrained efficiency. A generalization of the deferred acceptance process yields a stable assignment, but this outcome is not necessarily constrained efficient. We identify an easily verifiable sufficient condition for a stable assignment to be constrained efficient, which then leads to an algorithm to compute a constrained efficient assignment. Finally we illustrate practical applications of our framework and algorithm, including a widely studied matching problem with distributional constraints.
\end{abstract}

${ }^{*}$ Corresponding author.

†E-mail addresses: nae25@cam.ac.uk (A. Erdil), tkumano@ynu.ac.jp (T. Kumano) 
JEL classification: C78; D47; D61; D63

Keywords: Matching with indifferences; Efficiency; Stability; Substitutable priorities with ties

\section{Introduction}

Assigning indivisible goods to unit-demand agents is a classical economic problem. In many realistic contexts, monetary transfers (as would be in an auction) are ruled out for a variety of reasons, yet discrete resources are assigned via mechanisms which take recipients' preferences into account. Typically, each agent submits an ordinal preference ranking over the available goods, whereas each object comes with its exogenously fixed priorities which form the basis on which over-demanded objects are rationed. A widely used interpretation of respecting priorities is formalized by the pairwise stability property which is central to two-sided matching markets. For example, for an overdemanded school, students who live closer to the school can be given higher priority for that school; a criterion which can be expressed by a ranking over students. Respecting such priorities requires that a seat at school $s$ can be assigned to a student only when all students who have higher priority for $s$ are assigned a school which they weakly prefer to $s$. Note that this leads to a well-defined admission rule for the school (say with $q$ seats): given a set of applicants, those $q$ students who have the highest priority among those applicants are admitted. Abdulkadiroğlu and Sönmez (2003) show that these strict responsive admission rules operate like college preferences in Gale and Shapley's (1962) two-sided matching framework. In particular, the deferred acceptance algorithm of Gale and Shapley returns the unique constrained efficient assignment: the stable assignment which is preferred (weakly or strictly) by every student to all other stable assignments. However, not every plausible admission rule is a strict responsive rule. In particular there are two different and important extensions of the strict responsive framework. Substitutability, first introduced by Roth (1984a), is an ordinal analogue of Kelso and Crawford's (1982) gross substitutes condition in the absence of ties. In essence, it requires that whenever the set of applicants shrinks, those students who were admitted from the original set of applicants should continue to be admitted. Equivalently, whenever the set of applicants grows, those students rejected from the 
original set of applicants should still be rejected from the larger set of applicants. Strict responsive rules are substitutable, but as Echenique (2007) shows, the class of substitutable rules is much bigger than the class of responsive rules. Another direction to extend the domain of strict responsive rules is to allow for ties in the priority orders over students, while maintaining the responsiveness assumption. Commonly observed in school admission programs, such ties imply the admission rules are multivalued. An important consequence is that constrained efficient assignments are not unique any more, and the way ties are resolved in a deferred acceptance mechanism matters in terms of welfare as well as distribution of seats (Erdil and Ergin, 2008; Abdulkadiroğlu, Pathak and Roth, 2009). In this paper, we allow for ties and introduce the appropriate generalization of substitutability for multivalued admission rules. As such, the class of rules we explore will nest all of the above and provide the proper ordinal analogue of Kelso and Crawford's gross substitutes preferences without having to rule out ties.

Given any set of applicants, an admission rule is a correspondence that specifies which subsets of the applicants are admissible. For example, suppose a school with two seats is facing six equally qualified candidates consisting of two Asian, two black and two white students. A natural admission rule which gives 'diverse' cohorts priority over homogeneous cohorts would identify each mixed pair as an admissible subset of students from among the six applicants. Hence this school's admission rule will typically involve ties between several, but not all, possible subsets of the applicants. We define an admission rule to be substitutable if it is both admission monotonic (AM) and rejection monotonic (RM). Whenever the set of applicants becomes smaller, AM requires that for every admissible subset $S$ from the original set of applicants, there must be an admissible subset $S^{\prime}$ from the smaller set of applicants such that all those students in $S$ who are still among the applicants are in the admissible subset $S^{\prime}$. And whenever the set of applicants becomes larger, RM means for every rejectable subset $T$ (i.e., the complement of an admissible subset) from the original set of applicants, there must exist a rejectable subset $T^{\prime}$ of the larger set of applicants such that $T$ is contained in $T^{\prime}$. In the absence of ties, $\mathrm{AM}$ and RM are equivalent and boil down to the standard substitutability condition. However, neither of them implies the other when we allow for ties. Together, these two conditions are critical in ensuring that our Modified Deferred Acceptance (MDA) process constructively establishes the existence of a stable assignment (Proposition 2). 
Our framework allows modeling admission rules which do not necessarily fit into earlier formulations of priorities. Recall the example of a two-seat school facing six equally qualified applicants $a_{1}, a_{2}, b_{1}, b_{2}, w_{1}, w_{2}$, where letters indicate the applicants' types (Asian, black, and white, respectively). Prioritizing diverse cohorts over homogeneous cohorts, but otherwise staying indifferent between sets can be summarized by a transitive order $\succsim$ over pairs of students given by

$$
\left\{a_{*}, b_{*}\right\} \sim\left\{a_{*}, w_{*}\right\} \sim\left\{b_{*}, w_{*}\right\} \quad \succ \quad\left\{a_{1}, a_{2}\right\} \sim\left\{b_{1}, b_{2}\right\} \sim\left\{w_{1}, w_{2}\right\} .
$$

Note that this plausible and simple priority order $\succsim$ cannot be captured within the well-studied responsive framework, because if $\succsim$ were responsive $\AA^{1}$, we would necessarily have

$$
\left\{a_{1}, b_{1}\right\} \succ\left\{a_{1}, a_{2}\right\} \quad \Rightarrow \quad b_{1} \succ a_{2}
$$

and

$$
\left\{a_{2}, b_{2}\right\} \succ\left\{b_{1}, b_{2}\right\} \quad \Rightarrow \quad a_{2} \succ b_{1},
$$

yielding a contradiction. In Section 5.1, we expand on this example to combine more general distributional constraints with priorities that can be expressed as an order over the set of students (such as those based on walk-zone, sibling, or test-score). By showing substitutability of such priorities, we can appeal to the MDA process to find a stable assignment (Proposition 4). Importantly, the outcome of MDA is typically constrained inefficient, i.e., Pareto dominated by another stable assignment. We show (Proposition 3) that we can detect any such inefficiency by checking whether the assignment admits a potentially-stable improvement cycle (PSIC): one of our key constructions. PSIC is similar in spirit to Erdil and Ergin's (2008) stable improvement cycles (SIC), but functions differently in a number of ways. Unlike a SIC, a PSIC allows multiple students from the same school to move in the same cyclic trade, and such simultaneous moves do not necessarily preserve stability. Still, in the context of assignment with distributional constraints, we circumvent this issue and go on to establish an algorithm based on PSIC which indeed finds a constrained efficient assignment in polynomial time (Corollary 1).

Another class of applications are directly motivated by admission rules in two real-life contexts: admissions at the University of Cambridge, and course allocation in various

\footnotetext{
${ }^{1}$ Following Roth (1985), $\succsim$ is called responsive if for every $T \subset N$ and $i, j \notin T$, we have $T \cup\{i\} \succsim$ $T \cup\{j\} \Leftrightarrow i \succsim j$.
} 
Japanese universities. Deferring the descriptions of the actual procedures to the Appendix, we model the essential features of these mechanisms as follows: multiple referees in an admissions committee take turns to admit students from a given pool of applicants. Each referee $i$ has her own ranking $\succsim_{i}$ over the set of students. The referees can access the admissions files whenever they like, and until reaching a capacity constraint, admit as many students as they wish from the pool of remaining files according to their own ranking. If we were to fix the order in which the referees make decisions, and the numbers of students chosen in each decision, the admissions procedure would boil down to fixing a precedence order in Kominers and Sönmez's (2016) model of matching with slotspecific priorities. The workings of the above admissions committee, however, refrain from fixing such an order, and therefore implies a multivalued admission rule. While it is fairly straightforward to establish substitutability of this rule (thanks to Kominers and Sönmez' results), finding a constrained efficient assignment can be a significant challenge. We show that our PSIC process solves this problem (Corollary 2).

In Section 2, we introduce the model including the definition of substitutable priorities with ties before we formalise what we mean by respecting priorities. In Section 3 , we describe the modified deferred acceptance (MDA) process and show that it results in a stable assignment. In Section 4, we explain why constrained efficiency is a natural welfare benchmark, point out that the outcome of MDA is typically constrained inefficient, formally define a potentially-stable improvement cycle (PSIC), and establish its connection with constrained inefficiency. In Section 5, we study, in detail, two applications where constrained efficiency of an assignment is equivalent to lack of PSICs, and design a polynomial time algorithm which computes a constrained efficient assignment. Section 6 concludes.

\section{Preliminaries}

Let $N$ be a set of students, and $X$ be a set of schools. There are $q_{x}$ seats at school $x$, for $x \in X$. Each student can either be assigned to a school, or stay unassigned, and has strict preferences over these alternatives. Student $i$ 's preferences are denoted by a linear order $R_{i}$ over $X \cup\{i\}$, where being assigned to $i$ stands for staying unassigned (which can also be interpreted as getting one's outside option). $P_{i}$ denotes the strict part of $R_{i}$. 
Each school $x$ is endowed with its own priorities that help determine who can be admitted to that school. Formally, priorities are captured by an admission rule defined as follows. Given a set $S$ of applicants, a subset $S^{\prime}$ is called an admissible subset of $S$ if $S^{\prime}$ can be the set of admitted students. An admission rule associates to each set $S$ of students the collection of admissible subsets of $S$. If $\mathcal{A}_{x}$ is the admission rule for school $x$, then each $S^{\prime} \in \mathcal{A}_{x}(S)$ is considered to be of highest priority among all possible subsets of $S$. A priority structure $\mathcal{A}$ is a vector of admission rules: $\left(\mathcal{A}_{x}\right)_{x \in X}$. We call $\mathcal{A}$ acceptant if for each $x \in X$, for each $S \subseteq N$, and for each $S^{\prime} \in \mathcal{A}_{x}(S)$ we have $\left|S^{\prime}\right|=\min \left\{|S|, q_{x}\right\}$.

An admission rule is called strict if it associates to each set $S \subseteq N$ a unique subset of $S$. With a slight abuse of notation, for strict admission rules $\mathcal{A}_{x}$, we will write $\mathcal{A}_{x}(S)=S^{\prime}$ whenever $\mathcal{A}_{x}(S)=\left\{S^{\prime}\right\}$. A strict admission rule $\mathcal{A}_{x}^{\prime}$ is a tie-breaking of $\mathcal{A}_{x}$ if $\mathcal{A}_{x}^{\prime}(S) \in \mathcal{A}_{x}(S)$ for every $S \subseteq N$.

In order to have a well-defined notion of "an admissible subset having higher priority than those subsets which are not admissible" an admission rule must be consistent in the following sense: (1) given a set $S$ of applicants, if a subset $A$ is admissible but another subset $B$ is not, then $B$ is never an admissible subset of $S^{\prime}$ which also contains $A ;(2)$ given a set $S$ of applicants, if subsets $A$ and $B$ are both admissible, then they are either both admissible or both not admissible whenever they are both contained in a set $S^{\prime}$ of applicants. Formally, $\mathcal{A}_{x}$ being consistent means: (1) If there exists $S \subseteq N$ such that $A \cup B \subseteq S$ with $A \in \mathcal{A}_{x}(S)$ and $B \notin \mathcal{A}_{x}(S)$, then whenever $A \cup B \subseteq S^{\prime}$, we have $B \notin \mathcal{A}_{x}\left(S^{\prime}\right)$; and (2) if there exists $S$ such that $A, B \in \mathcal{A}_{x}(S)$, then whenever $A \cup B \subseteq S^{\prime}$, we have $A \in \mathcal{A}_{x}\left(S^{\prime}\right)$ if and only if $B \in \mathcal{A}_{x}\left(S^{\prime}\right)$. A reflexive and transitive (but possibly incomplete) order $\succsim_{x}$ over subsets of $N$ is consistent with $\mathcal{A}_{x}$ if the following two conditions hold: (1) if $A \cup B \subseteq S, A \in \mathcal{A}_{x}(S)$ and $B \notin \mathcal{A}_{x}(S)$ for some $S \subseteq N$, then $A \succ_{x} B$; (2) if $A \cup B \subseteq S, A \in \mathcal{A}_{x}(S)$ and $B \in \mathcal{A}_{x}(S)$ for some $S \subseteq N$, then $A \sim_{x} B$. There are many orders consistent with a given admission rule $\mathcal{A}_{x}$. However, for every such $\succsim_{x}$, we have $\mathcal{A}_{x}(S)=\left\{S^{\prime} \subseteq S \mid S^{\prime} \succsim_{x} T^{\prime}\right.$ for each $\left.T^{\prime} \subseteq S\right\}$ for each $S \subseteq N$.

Given an admission rule $\mathcal{A}_{x}$, it is helpful to define its rejection rule $\mathcal{R}_{x}$, which associates to each $S \subseteq N$, the family of subsets of $S$ each of which can be the set of rejected students when $S$ is the set of applicants. That is,

$$
\mathcal{R}_{x}(S)=\left\{S^{\prime \prime} \subseteq S \mid S^{\prime \prime}=S \backslash S^{\prime} \text { for some } S^{\prime} \in \mathcal{A}_{x}(S)\right\}
$$


Each $S^{\prime \prime} \in \mathcal{R}_{x}(S)$ is called a rejectable subset of $S$.

We say $\mathcal{A}_{x}$ is admission monotonic (AM) if given any $S, T \subseteq N$ with $S \subseteq T$ and $T^{\prime} \in \mathcal{A}_{x}(T)$, we have $T^{\prime} \cap S \subseteq S^{\prime}$ for some $S^{\prime} \in \mathcal{A}_{x}(S)$. In a similar spirit, $\mathcal{A}_{x}$ is rejection monotonic (RM) if given any $S, T \subseteq N$ with $S \subseteq T$ and $S^{\prime \prime} \in \mathcal{R}_{x}(S)$, we have $S^{\prime \prime} \subseteq T^{\prime \prime}$ for some $T^{\prime \prime} \in \mathcal{R}_{x}(T)$. When there are no ties, both $\mathcal{A}_{x}$ and $\mathcal{R}_{x}$ are strict, and AM is equivalent to RM. However neither implies the other when there are ties.2 We call an admission rule substitutable if it is both admission monotonic and rejection monotonic. Moreover, we say a priority structure $\mathcal{A}$ is substitutable if $\mathcal{A}_{x}$ is substitutable for all $x \in X$.

For an intuitive explanation of the conditions AM and RM in a concrete example, consider a university admissions office determining who will be admitted from a given set $T$ of applicants. Suppose $T^{\prime}$ is the set of students who receive an offer conditional on successfully graduating from secondary school. Once the final exam grades are revealed, only a subset $S$ of applicants meet this condition for admission. The admission rule being admission monotonic ensures that those students who had received a conditional offer and subsequently met the condition $\left(T^{\prime} \cap S\right)$ can be among the admitted students if the admission rule is applied directly to the set $S$ of eligible students. In an alternative scenario, suppose the subset $S^{\prime \prime}$ of eligible applicants $S$ have ultimately been rejected. Of those applicants who failed to graduate, some successfully appeal against their exam grades, and consequently get their names back in the set of eligible applicants. The admission rule being rejection monotonic ensures that those students rejected from the original set of eligible applicants can still be rejected from the new (and larger) set of eligible applicants.

The class of admission rules which satisfy substitutability covers various environments studied in the literature ${ }^{3}$ For example, hospitals' strict responsive preferences

${ }^{2}$ When there are no ties, AM reduces to the definition of substitutability in Roth and Sotomayor (1990). In the context of matching with contracts where doctors have strict preferences over contracts, Hatfield and Milgrom's (2005) substitutability formulation is similar to RM: the set of rejected contracts expands whenever the set of available contracts expands. Remark 1 in the Online Appendix proves the equivalence [independence] of AM and RM in the absence [presence] of ties.

${ }^{3}$ Péter Biró and Paul Harrenstein brought to our attention Sotomayor's (1999) definition of substitutability in the context of preferences with ties: player $y$ has substitutable preferences if (i) for all $S^{\prime} \in C h_{y}(F \cup G)$ there is some $S \in C h_{y}(F)$ such that $S^{\prime} \cap F \subseteq S$ and (ii) for all $S \in C h_{y}(F)$ there 


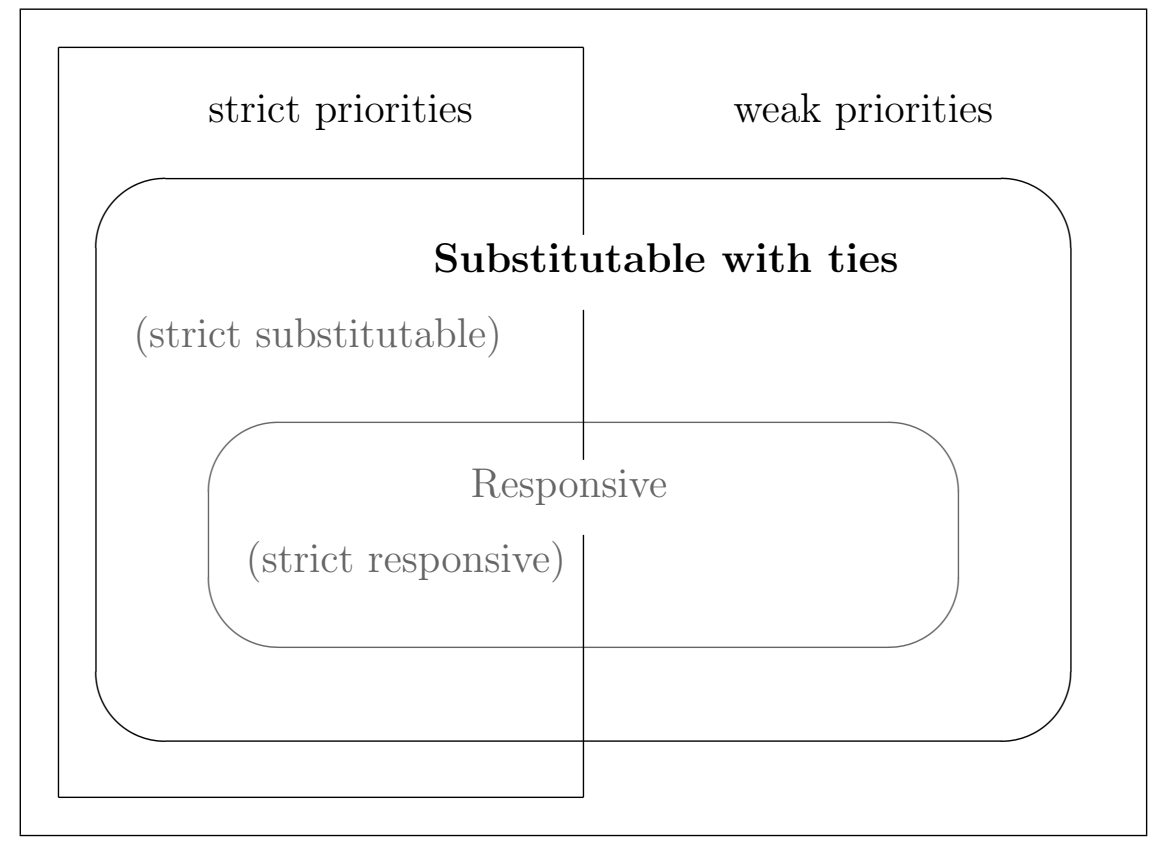

Figure 1: The class of substitutable priorities with ties is strictly larger than that of both strict substitutable priorities and responsive priorities with ties.

over sets of doctors (Roth, 1984b), the school choice formulation of Abdulkadiroğlu and Sönmez (2003), school priorities with ties (Erdil and Ergin, 2008), and strict substitutable firm preferences in Roth (1984a) are all special cases.

An assignment (i.e., a matching) $\mu$ is a function $\mu: N \rightarrow X \cup N$ such that $\mu(i) \in X \cup\{i\}$ for all $i \in N$, and $\left|\mu^{-1}(x)\right| \leq q_{x}$ for all $x \in X$. In words, each student is matched with a school or her outside option; and each school $x$ is matched with at most $q_{x}$ students.

Suppose a group of students prefer $x$ to their respective matched schools, and contest the admissions outcome at school $x$. Let the school's admission rule be applied to the combined set of currently matched students and those who contest the outcome. If the original subset of admitted students is not an admissible subset of this combined set of is some $S^{\prime} \in C h_{y}(F \cup G)$ such that $S^{\prime} \cap F \subseteq S$. We show in Remark 2 in the Online Appendix that we can replace rejection monotonicity with the following condition: given any $S \subseteq T$, and $S^{\prime} \in \mathcal{A}_{x}(S)$, we have $T^{\prime} \cap S \subseteq S^{\prime}$ for some $T^{\prime} \in \mathcal{A}_{x}(T)$. By doing so, we establish the equivalence between our formulation and Sotomayor's (1999) formulation. 
applicants, i.e., if there is a new group of students who demand $x$, while having strictly higher priority than the set originally admitted, then the original assignment violates priorities. Respecting priorities (also known as group stability) means robustness to claims by such groups, and is formally defined as:

- for each $i \in N$ we have $\mu(i) R_{i} i$, and

- for each $x \in X$ and $S \subseteq N$ such that $\mu^{-1}(x) \subseteq S \subseteq\left\{i \in N \mid x R_{i} \mu(i)\right\}$ we have $\mu^{-1}(x) \in \mathcal{A}_{x}(S)$.

A weaker requirement is robustness to claims by individuals. Formally, an assignment $\mu$ is pairwise stable if $\mu(i) R_{i} i$ for all $i \in N$; and for each $x \in X$ and $j \in N$ such that $x P_{j} \mu(j)$ we have $\mu^{-1}(x) \in \mathcal{A}_{x}\left(\mu^{-1}(x) \cup\{j\}\right)$. When all schools have substitutable and consistent admission rules, the two notions of stability above are equivalent.

Proposition 1 Given a substitutable and consistent priority structure, an assignment respects priorities (i.e., is group stable) if and only if it is pairwise stable.

Thus the equivalence of pairwise stability and group stability holds in a significantly more general environment than what was shown in earlier literature. An obvious convenience of this result is that verifying that an assignment respects group priorities can be reduced to simply checking pairwise stability. From this point onward, for brevity we will use the term stable to capture the notion of respecting priorities, i.e., group stability.

\section{The modified deferred acceptance process}

We establish the existence of a stable assignment via a constructive process in the spirit of Gale and Shapley's Deferred Acceptance (DA) Algorithm. However, the extension to our more general domain is not immediate. First, the priorities we consider allow ties, and therefore, the admission rules are multivalued. Gale and Shapley (1962) note in their original paper that when there are ties in rankings, arbitrarily breaking them and continuing with their algorithm will result in a pairwise stable assignment 4 This

\footnotetext{
${ }^{4}$ This fact has since been invoked in Erdil and Ergin (2008) and Abdulkadiroğlu, Pathak and Roth (2009). They maintain the environment where priorities can be expressed via rankings over individuals,
} 
convenience does not survive into our environment. Namely, as we illustrate in Remark 3 in the Online Appendix, breaking ties arbitrarily, and then applying DA might result in an assignment which is not stable. Moreover, a substitutable admission rule does not necessarily have any substitutable tie-breaking (Remark 4).

We modify the deferred acceptance process to impose a "monotonicity condition" on the way our process selects from multivalued correspondences. More concretely, we will make sure that those students who were rejected by school $x$ in any round of the process continue to be rejected in later rounds.

\section{Modified Deferred Acceptance Process (MDA)}

Round 1: All students apply to their favorite schools. For each school $x$, if $A_{x}^{1}$ is the set of applicants, select a set $S_{x}^{1}$ in $\mathcal{A}_{x}\left(A_{x}^{1}\right)$ to be the set of temporarily admitted students. The rest of the applicants, denoted $Z_{x}^{1}=$ $A_{x}^{1} \backslash S_{x}^{1}$, are rejected.

For $t \geq 2$,

Round $t$ : Those who were rejected in round $t-1$ apply to their next favorite school. For each school $x$, if $A_{x}^{t}$ is the set of all students who have applied to $x$ so far, select a set of temporarily admitted students $S_{x}^{t} \in \mathcal{A}_{x}\left(A_{x}^{t}\right)$ such that $Z_{x}^{t-1} \subseteq A_{x}^{t} \backslash S_{x}^{t}$. The rest of the applicants, denoted $Z_{x}^{t}=A_{x}^{t} \backslash S_{x}^{t}$, are rejected.

When every student is either matched with a school or has been rejected by all schools in her list, the process ends.

Note that the above process does not specify how it selects the temporarily admitted students $S_{x}^{t}$ when there is more than one subset $S$ of $\mathcal{A}_{x}\left(A_{x}^{t}\right)$ such that $Z_{x}^{t-1} \subseteq A_{x}^{t} \backslash S$. Before we turn to specifying such selections, we establish the main property of the above class of algorithms independent of such selection.

Proposition 2 Given a substitutable priority structure $\mathcal{A}$, the outcome of the Modified Deferred Acceptance Process is stable.

so the existence of stable assignments in the presence of ties follows from Gale and Shapley (1962). 
Our ability to select a set of temporarily admitted students $S_{x}^{t} \in \mathcal{A}_{x}\left(A_{x}^{t}\right)$ such that $Z_{x}^{t-1} \subseteq A_{x}^{t} \backslash S_{x}^{t}$ relies on $\mathcal{A}_{x}$ being rejection monotonic. Selecting with this property ensures that those applicants rejected in any round continue to be rejected in later rounds. In particular, if all those students who prefer $x$ to their final match were to re-apply to $x$, they can all be rejected as long as all those matched with $x$ continue to be in the pool of applicants. In turn, $\mathcal{A}_{x}$ being acceptance monotonic ensures that the admitted set at school $x$ is one of the highest priority sets whenever a group of students who prefer $x$ to their final match contest the assignment and instead seek to be matched with $x$. In other words, whichever subset of students who prefer $x$ to their final match re-apply to $x$, those who are matched to $x$ can still form an admitted set. The above two properties, together, ensure that the outcome of MDA is stable. The details of the argument are in the Appendix.

MDA is closely related to Kelso and Crawford's (1982) salary adjustment process $(S A P)$. Their classic model of firm-worker matching involves workers whose utilities depend on the firm for which they work and the salary they get. These utilities are strictly increasing in salary. The firm's preferences over sets of workers are assumed to satisfy the so-called gross substitutes condition which "requires that all workers be (weak) gross substitutes to each other, in the sense that increases in other workers' salaries can never cause a firm to withdraw an offer from a worker whose salary has not risen." To be more precise, given a salary vector (which specifies a salary for each potential worker), the firm has an "offer correspondence" which satisfies the above condition. MDA is a student-proposing process during which the opportunity sets of schools expand. In contrast, in Kelso and Crawford's firm-proposing SAP, workers' opportunity sets get (weakly) better at each round. Since the offer correspondence specifies multiple subsets of workers, SAP needs to select a subset at each round. And these selections are made so that "any offer made by firm $j$ in round $t-1$ that was not rejected must be repeated in round $t$." The proper analogue of this property in our student-proposing setting is the condition $Z_{x}^{t-1} \subseteq A_{x}^{t} \backslash S_{x}^{t}$ which says those students rejected in round $t-1$ should continue to be rejected in round $t$.

The MDA process we described above is flexible as to how we select $S_{x}^{t}$ from $\mathcal{A}_{x}\left(A_{x}^{t}\right)$ except for ensuring $Z_{x}^{t-1} \subseteq A_{x}^{t} \backslash S_{x}^{t}$. By fixing these choices deterministically (e.g., by appealing to an ex ante fixed ranking over sets) or randomly, we obtain an MDA mech- 
anism. Even though a tie-breaking rule (either an ex ante order over sets or a randomization protocol) can be fixed independent of revealed preferences, the way in which the MDA process unfolds might, in principle, be dependent on the order in which applications are made. In particular, it is yet unknown whether there is a strategy-proof MDA mechanism.

\section{Efficiency and constrained efficiency}

Since the problems we have in mind revolve around assigning indivisible resources (e.g., school seats) to unit-demand agents (e.g., students), the most basic efficiency criterion requires not wasting resources. In particular, non-wastefulness means an unused school seat cannot be denied to any student. Formally, an assignment $\mu$ is called non-wasteful if no student prefers a school with unassigned seats to her match. That is, $\left|\mu^{-1}(x)\right|=q_{x}$ whenever there exists $i \in N$ such that $x P_{i} \mu(i)$. It is not hard to see that if $\mu$ is stable under an acceptant priority structure $\mathcal{A}$, then it is non-wasteful. There is, of course, more to efficiency, and Pareto efficiency is the standard welfare benchmark in assignment problems. An assignment $\mu^{\prime}$ Pareto dominates another assignment $\mu$ if $\mu^{\prime}(i) R_{i} \mu(i)$ for all $i \in N$, and $\mu^{\prime}(j) P_{j} \mu(j)$ for some $j \in N$. An assignment is efficient if it is not Pareto dominated by any assignment. Finally, an assignment is constrained efficient if it is stable and is not Pareto dominated by any other stable assignment.

When all schools have strict responsive priorities, Gale and Shapley (1962) show that the DA algorithm returns the unique constrained efficient assignment. However, this assignment may not be efficient. In other words, given priorities and preferences, it is possible that none of the stable assignments are efficient. In his study of this conflict between efficiency and stability, Ergin (2002) establishes a complete characterization of strict responsive priority structures for which the constrained efficient assignment is efficient. A key conclusion of his analysis is that, when allocating on the basis of priorities, for the conflict between stability and efficiency to not arise, all schools must have "sufficiently similar" priority rankings 5 $^{5}$ Generalizations of this finding to weak

\footnotetext{
${ }^{5}$ Ergin (2002) shows that for strict responsive priorities the unique constrained efficient assignment is efficient for all preference profiles if and only if the priority structure satisfies a property he calls acyclicity, which can be interpreted as all schools having "sufficiently similar" priority rankings. For
} 
responsive priorities also find conditions on priorities which naturally admit the same interpretation: if priorities differ from one school to another, we cannot guarantee stability and efficiency at the same time.$^{6}$ One clear message of this line of research is that the conditions under which we can escape the conflict between efficiency and stability are highly restrictive. When we turn to the much more general environment of substitutable priorities with ties, even having identical priorities across all schools is not enough to avoid this conflict. We illustrate this possibility in the following example.

Example. Suppose there are two schools $x$ and $y$ with two seats each, and six applicants, $a_{1}, a_{2}, b_{1}, b_{2}, w_{1}, w_{2}$, where $a, b, w$ stand for Asian, black and white, respectively. Both schools prioritize mixed pairs over same type pairs. Moreover, between two mixed pairs, those with more minority ( $a$ or $b$ ) students are given priority. Hence their priorities over student pairs can be summarized as

$$
\left\{a_{*}, b_{*}\right\} \succ\left\{a_{*}, w_{*}\right\} \sim\left\{b_{*}, w_{*}\right\} \succ\left\{a_{1}, a_{2}\right\} \sim\left\{b_{1}, b_{2}\right\} \sim\left\{w_{1}, w_{2}\right\} .
$$

Suppose all students find both schools acceptable. Moreover type- $a$ students prefer $x$ to $y$, whereas type- $b$ students prefer $y$ to $x$ :

$$
x P_{a} y P_{a} a \quad, \quad y P_{b} x P_{b} b \quad, \quad x P_{w} w \text { and } y P_{w} w .
$$

Then there are exactly four stable assignments, and at each such assignment both schools have one type- $a$ and one type- $b$ student. All four assignments can be Pareto improved by assigning both type- $a$ students to $x$, and both type- $b$ students to $y$. However, the resulting Pareto efficient assignment would not be stable due to demand by $w_{1}$ and $w_{2}$.

an explicit example of an Ergin cycle, let $i, j, k$ be three students, and $x, y$ be two schools with one seat each such that $i \succ_{x} j \succ_{x} k \succ_{y} i$. If students' preferences are $y P_{i} x, x P_{j} j P_{j} y$ and $x P_{k} y$, the only constrained efficient assignment is $(i x, j j, k y)$ which is not efficient, because it is Pareto dominated by $(i y, j j, k x)$, and the latter assignment is not stable due to student $j$ 's demand for school $x$. The source of inefficiency in this example is due to schools $x$ and $y$ ranking students $i$ and $k$ sufficiently differently: compared with $y$ 's ranking, $x$ swaps $i$ and $k$, and inserts another student $j$ in between them.

${ }^{6}$ For responsive priorities with ties, typically there are multiple constrained efficient assignments. In this larger domain, Erdil and Ehlers (2010) characterize priority structures for which all constrained efficient assignments are efficient. Han (2018), instead, establishes a characterization of priority structures for which a stable and efficient assignment is guaranteed to exist. The conditions obtained in these two papers boil down to Ergin's acyclicity when there are no ties. 
Hence, in contrast with Ehlers and Erdil (2010) and Han (2018), identical priorities for schools do not ensure efficiency.

In Remark 7 in the Online Appendix we present a necessary and sufficient condition on priority structures so that all constrained efficient assignments are efficient. A more permissive condition would require that at least one constrained efficient assignment is efficient for all preference profiles. While we have not established a condition of that sort, we know that such a condition would necessarily be at least as restrictive as that of Han (2018) which is more demanding than Ergin acyclicity. In particular, even identical priorities like those illustrated in the above example would be ruled out. Since most priority structures in practice will fail the aforementioned acyclicity condition and its various generalizations, respecting priorities (defined as stability) will imply that our natural welfare benchmark and objective is assigning school seats as efficiently as possible subject to stability. Thus our focus is on constrained efficiency.

Roth (1984a) shows that under strict substitutable priorities the DA algorithm returns the unique constrained efficient assignment. However, DA does not lend itself to an obvious solution when there are ties. In fact, for substitutable priorities, arbitrarily resolving the ties and proceeding with DA might even result in an unstable assignment.77 The MDA process is careful in the way it breaks ties to make sure the final assignment is stable, but this assignment is not necessarily constrained efficient 8 Is there a practical way to determine whether a stable assignment admits a stability preserving Pareto improvement? And is there a practical way to discover such improvements so that we can indeed reach a constrained efficient outcome? In exploring the nature of such improvements, Erdil and Ergin's (2008) stable improvement cycles might seem like a good candidate. While we build on their ideas, we will also explain why their constructions and results do not readily extend to the domain of substitutable priorities. Consequently, we will need to define a new notion of an improvement cycle for our more general setting.

Given stable $\mu$, if $j$ were to disappear, who could be taking $j$ 's seat at school $\mu(j)$ while preserving stability of the assignment? If a student $i$ replaces $j$, the new set of

\footnotetext{
${ }^{7}$ Remark 3 in the Online Appendix illustrates this possibility.

${ }^{8}$ Ehlers (2007) shows how arbitrary tie-breaking leads to constrained inefficiency when priorities are responsive with ties. Since our environment subsumes that of responsive priorities, the same inconvenience automatically extends to our setting.
} 
students assigned to $\mu(j)$ must be an admissible set in the face of those who would like to be replacing $j$ at $\mu(j)$. To formalize this idea, let $\mathcal{E}_{j}^{\mu}$ stand for the set of students who envy $j$ at assignment $\mu$, that is, $\mathcal{E}_{j}^{\mu}=\left\{i \mid \mu(j) P_{i} \mu(i)\right\}$. Then, the set of students who can replace student $j$ at $\mu$ is defined as

$$
E_{j}^{\mu}=\left\{i \in \mathcal{E}_{j}^{\mu} \mid\{i\} \cup\left[\mu^{-1}(\mu(j)) \backslash\{j\}\right] \in \mathcal{A}_{\mu(j)}\left(\mathcal{E}_{j}^{\mu} \cup\left[\mu^{-1}(\mu(j)) \backslash\{j\}\right]\right)\right\}
$$

Note that $j \notin E_{j}^{\mu}$, and $E_{j}^{\mu}$ is not necessarily a singleton. Moreover if all students not matched with $\mu(j)$ prefer their current match to $\mu(j)$, then $E_{j}^{\mu}$ is empty. A potentiallystable improvement cycle (PSIC) consists of distinct students $i_{0}, i_{1}, \ldots, i_{n-1}, i_{n}=i_{0}$ such that $i_{\ell} \in E_{i_{\ell+1}}^{\mu}$ for all $\ell=0, \ldots, n-1$. We denote such a cycle by $i_{0} \rightarrow i_{1} \rightarrow$ $\cdots \rightarrow i_{n-1} \rightarrow i_{0}$. To carry out this PSIC means replacing $\mu$ with assignment $\nu$ where $\nu\left(i_{\ell}\right)=\mu\left(i_{\ell+1}\right)$ for each $\ell=0, \ldots, n-1$, and $\nu(i)=\mu(i)$ for each $i \notin\left\{i_{0}, i_{1}, \ldots, i_{n-1}\right\}$. We say this PSIC preserves stability if $\nu$ is stable. Unlike a stable improvement cycle in Erdil and Ergin (2008), PSIC allows a school to appear more than once in a cycle. $\square^{9}$

Proposition 3 Given an acceptant and substitutable priority structure, if a stable assignment does not admit a PSIC, then it is constrained efficient.

Given a stable assignment, determining whether it admits a PSIC is a simple exercise of searching for a cycle in a directed graph. If the assignment does not admit a PSIC, then the above proposition concludes that it must be constrained efficient. However, the converse does not necessarily hold: a constrained efficient assignment might admit a PSIC. Put differently, a PSIC does not necessarily preserve stability 10 When searching for a constrained efficient outcome, if the PSIC we discover preserves stability, then carrying it out yields a new stable assignment which Pareto improves the assignment. Without knowing that the converse of Proposition 3 holds, it is not clear how

\footnotetext{
${ }^{9}$ Their definition of a stable improvement cycle (SIC) does not capture all improvement cycles that preserve stability in our environment. Remark 6 in the Online Appendix illustrates a constrained inefficient assignment which admits a PSIC but not a SIC.

${ }^{10}$ Remark 5 illustrates the possibility that a constrained efficient assignment admits a PSIC (which means this PSIC does not preserve stability), hence the qualifier "potentially" in PSIC. The construction of a PSIC ensures that each student can replace the one she is pointing at without violating stability. However, when the cycle involves two students at the same school, the two of them being replaced by two new students might well destabilize the assignment.
} 
many PSICs we need to explore before we can conclude that we have indeed reached a constrained efficient assignment. And exhausting all possible PSICs might be computationally demanding (as in requiring exponential time). In general, we do not know if there is a polynomial time algorithm which computes a constrained efficient assignment. When constrained efficiency is equivalent to lack of PSIC, i.e., when the converse of the above proposition also holds, we can indeed design practical (and in particular polynomial time) algorithms to compute a constrained efficient assignment. We do not have a characterization or a suggestive description of all environments for which the converse of Proposition 3 holds. Instead, in the next section, we present two applications for which such an equivalence (formally expressed in Propositions 5 and 67 indeed holds. Then we describe the PSIC algorithm, and explain why it is polynomial time.

\section{Applications}

We present two classes of applications where our framework goes beyond the previously studied models in answering questions involving how ties are handled and how constrained efficiency can be recovered when arbitrary tie-breaking rules fail.

\subsection{Assignment with distributional constraints}

In this application, we generalize the idea of prioritizing diverse cohorts which we discussed in a simple example in the introduction. Namely, we formalize EDCR: our notion of allocating seats as evenly as possible across different types of recipients.

When the students can be classified into different types and the policy maker primarily cares about the distribution of types among those assigned to a particular school, a common modeling approach involves type-specific reserves ${ }^{11}$ To make things concrete, let $\mathcal{T}=\left\{\tau_{1}, \ldots, \tau_{m}\right\}$ be the set of types, and $\tau: N \rightarrow \mathcal{T}$ be a function such that $\tau(i)$ indicates student $i$ 's type. School $x$ has $q_{x}$ seats and its own vector of reserves

\footnotetext{
${ }^{11}$ This formulation of reserves is similar to the type-specific minimum quatas in Hafalir, Yenmez, and Yildirim (2013). See also Echenique and Yenmez (2015). Our key modeling difference is to allow for ties in priorities. By doing so, we can capture a richer space of admission rules. Moreover, by dealing with ties in a careful way, we are able to avoid potential welfare losses associated with arbitrarily reducing this richer environment to earlier models with strict priorities.
} 
$\boldsymbol{q}_{x}=\left(q_{x}^{\tau_{1}}, \ldots, q_{x}^{\tau_{m}}\right)$, where $\sum_{k=1}^{m} q_{x}^{\tau_{k}} \leq q_{x}$. If these reserves add up $q_{x}$, we can interpret $\boldsymbol{q}_{x}$ as the target distribution ${ }^{12}$ of types at school $x$. School $x$ 's other priorities (e.g., walking-zone, sibling, etc.) are summarized by a weak order on $N$ which we will refer to as an exogenous priority ranking. In principle, each school may have its own exogenous priority ranking, but whenever it leads to no confusion, we will suppress this ranking's dependence on $x$, and will simply denote it by $\succsim^{e x o}$.

The interpretation for reserving $q_{x}^{\tau}$ seats for type- $\tau$ students is that the school should not assign any such seat to a student whose type is not $\tau$ unless there are less than type- $\tau$ students interested in this school. Of course all seats at this school are identical from the perspective of the students, and what matters is the number of reserved seats. So respecting reserves requires admitting all type- $\tau$ students if the number of type- $\tau$ applicants is less than $q_{x}^{\tau}$, and admitting at least $q_{x}^{\tau}$ type- $\tau$ students otherwise. Formally, we say a set $S^{\prime} \subseteq S$ respects reserves in $S$ if for all $\tau$ in $\mathcal{T}$, we have $\left|S_{\tau}^{\prime}\right| \geq \min \left(\left|S_{\tau}\right|, q_{x}^{\tau}\right)$, where $S_{\tau}=\{s \in S: \tau(s)=\tau\}$. An admission rule $\mathcal{A}_{x}$ respects reserves (RR) if for all $S \subseteq N$, each $S^{\prime} \in \mathcal{A}_{x}(S)$ respects reserves in $S$. Note that this property does not put any restriction on how to assign seats which are not filled by their intended types. Neither does it suggest which type- $\tau$ students should get the seats reserved for their type. Hence an admission rule based purely on RR inevitably leads to vast numbers of subsets of applicants to be deemed admissible.

By appealing to responsiveness, the exogenous priority order $\succsim^{\text {exo }}$ can provide further guidance in choosing between sets that respect reserves. To put it more concretely, we say $\mathcal{A}_{x}$ is reserve constrained responsive (RCR) to $\succsim^{\text {exo }}$ if it respects reserves and there exists a reflexive and transitive order $\succsim_{x}$ on subsets of $N$ which is consistent with $\mathcal{A}_{x}$ and satisfies the following property: whenever $|S|=q_{x}-1 ; s^{\prime}, s^{\prime \prime} \notin S$; and both $S \cup\left\{s^{\prime}\right\}$ and $S \cup\left\{s^{\prime \prime}\right\}$ respect reserves in $S \cup\left\{s^{\prime}, s^{\prime \prime}\right\}$,

$$
S \cup\left\{s^{\prime}\right\} \succsim_{x} S \cup\left\{s^{\prime \prime}\right\} \quad \Leftrightarrow \quad s^{\prime} \succsim^{e x o} s^{\prime \prime}
$$

If this exogenous order $\succsim^{\text {exo }}$ is strict, RCR refines an admission rule which otherwise is based purely on RR, and we obtain a strict admission rule which Echenique and Yenmez (2015) call "generated by reserves for priority". In practice, $\succsim^{\text {exo }}$ might involve ties (which, for example, is the case for the Boston School Choice System). How we

\footnotetext{
${ }^{12}$ Echenique and Yenmez (2015) call this "an ideal point".
} 
resolve the ties in $\succsim^{e x o}$ will have an impact on the resulting distribution of types. For example, consider a school with six seats and reserve vector $\boldsymbol{q}=\left(q_{a}, q_{b}, q_{w}\right)=(2,2,2)$ facing eight applicants four of whom are type- $a$, and the other four type- $b$. So the two seats reserved for type- $w$ are not demanded by any type- $w$ students, and therefore need to be assigned among other types. Depending on the tie-breaking, the school can end up with either of the three type distributions: $(4,2,0),(3,3,0)$, or $(2,4,0)$. In the absence of further criteria to promote one distribution over another, we will ask how a "symmetric" treatment of types can be implemented. This idea of symmetry follows the spirit of the example we gave in the introduction: surplus seats (seats not reserved for any type or not filled by their intended types) shall be rationed evenly among over-subscribed types 13 So, in the example above, the two seats intended for (and not claimed by) type- $w$ students should be allocated evenly between type- $a$ and type- $b$. As we illustrated in the introduction, such an allocation rule cannot possibly be responsive. Below, we first formalize this rule, then show that it is substitutable, and finally develop an algorithm to compute a constrained efficient assignment.

Given a school $x$ with a reserve vector $\boldsymbol{q}_{x}$, define the distance ed $\left(\boldsymbol{q}_{x}, S\right)$ between $\boldsymbol{q}_{x}$ and set $S$ of students as

$$
\operatorname{ed}\left(\boldsymbol{q}_{x}, S\right)=\sum_{\tau \in \mathcal{T}}\left(\left|S_{\tau}\right|-q_{x}^{\tau}\right)^{2}
$$

We say an admission rule $\mathcal{A}_{x}$ evenly distributes (ED) surplus seats if there exists a reflexive and transitive order $\succsim_{x}$ on subsets of $N$ which is consistent with $\mathcal{A}_{x}$ and satisfies the following property: for every $S, S^{\prime}$ such that $|S|=\left|S^{\prime}\right|=q_{x}$,

$$
\operatorname{ed}\left(\boldsymbol{q}_{x}, S\right) \leq \operatorname{ed}\left(\boldsymbol{q}_{x}, S^{\prime}\right) \quad \Leftrightarrow \quad S \succsim_{x} S^{\prime}
$$

\footnotetext{
${ }^{13} \mathrm{RCR}$ implies that when students of type- $\tau$ are being matched, they are initially assigned to seats reserved for their type with the highest $\succsim^{e x o}$ priority students being assigned first. That means the competition for surplus seats is among those students who are lower in $\succsim^{e x o}$. Moreover, in our formulation of a school's admission rule, the goal is to achieve a distribution of types as close to the school's "target distribution" as possible. Subject to achieving that objective, other priorities (summarized by $\succsim^{\text {exo }}$ ) help determine who is assigned the over-demanded schools. Dur, Kominers, Pathak and Sönmez (2018) show that this particular design choice has significant distributional implications both in theory and practice. In general, there is a conflict between different priorities whether they are motivated by promoting diversity, walk-zone attendance, or rewarding higher achieving students. We are not addressing this trade-off or alternative ways of reconciling these competing objectives.
} 
First note that ED implies RR. That is, ED implies a refinement of an admission rule which only satisfies RR. However, the discrete nature of our problem will still lead to ties between several sets. Taking ED as the leading priority, a further refinement can be achieved by appealing, again, to an exogenous priority order $\succsim^{e x o}$ over the set of students. Formally, we say $\mathcal{A}_{x}$ is ED-constrained responsive (EDCR) to $\succsim^{\text {exo }}$ if there exists a reflexive and transitive order $\succsim_{x}$ on subsets of $N$ which is consistent with $\mathcal{A}_{x}$ and satisfies:

(i) whenever $|S|=\left|S^{\prime}\right|=q_{x}$, we have $\operatorname{ed}\left(\boldsymbol{q}_{x}, S\right)<\operatorname{ed}\left(\boldsymbol{q}_{x}, S^{\prime}\right) \Rightarrow S \succ_{x} S^{\prime}$, and

(ii) whenever $\left|S \cup\left\{s^{\prime}\right\}\right|=\left|S \cup\left\{s^{\prime \prime}\right\}\right|=q_{x}$ and $\operatorname{ed}\left(\boldsymbol{q}_{x}, S \cup\left\{s^{\prime}\right\}\right)=\operatorname{ed}\left(\boldsymbol{q}_{x}, S \cup\left\{s^{\prime \prime}\right\}\right)$,

$$
S \cup\left\{s^{\prime}\right\} \succsim_{x} S \cup\left\{s^{\prime \prime}\right\} \quad \Leftrightarrow \quad s^{\prime} \succsim^{\text {exo }} s^{\prime \prime}
$$

Proposition 4 An acceptant admission rule which satisfies EDCR is substitutable.

Thus, for such priority structures, MDA yields a stable assignment ${ }^{14}$ However, this stable assignment is not necessarily constrained efficient. Our first step is to provide a characterization of constrained efficient assignments. Using this characterization, we will next design an algorithm that solves the problem of finding a constrained efficient assignment for any given profile of preferences.

Proposition 5 Given an acceptant priority structure which satisfies EDCR, a stable assignment is constrained efficient if and only if it does not admit a potentially-stable improvement cycle.

A key step in proving the above proposition is to establish that the shortest PSIC preserves stability while Pareto improving the assignment. Deferring the details of the

\footnotetext{
${ }^{14}$ For this class of priority structures, it is actually possible to compute a stable assignment without using the terminology of substitutability or MDA. Given a set of applicants, first admit the highest ranked $q_{x}^{\tau}$ students of type $\tau$. If there are any seats and applicants remaining, then for each type $\tau$, take one student who has the highest priority among the remaining type- $\tau$ applicants. From this set of (at most $m$ ) students, accept them according to the ranking $\succsim^{e x o}$ up to the capacity of school $x$. Repeat this procedure until the capacity of $x$ is filled or the applicants are exhausted. We are grateful to the referee who suggested this alternative approach. Like MDA, this alternative approach typically fails to return a constrained efficient assignment.
} 
argument to the Appendix, we now use this finding to design an algorithm which always returns a constrained efficient assignment.

\section{Potentially-Stable Improvement Cycles Algorithm}

\section{Step 0:}

Run the $M D A$ process to obtain an initial assignment $\mu^{0}$.

\section{Step $t \geq 1$ :}

(t.a) Given $\mu^{t-1}$, let the students stand for the vertices of a directed graph, where for each pair of students $i$ and $j$, there is an edge $i \rightarrow j$ if and only if $i \in E_{j}^{\mu^{t-1}}$.

$(t . b)$ If there are any potentially-stable improvement cycles in this directed graph, select a shortest one, and carry out this cycle to obtain $\mu^{t}$, and go to step $(t+1 . a)$. If there is no such cycle, then return $\mu^{t-1}$ as the outcome of the algorithm.

Corollary 1 If priorities satisfy EDCR, then the PSIC algorithm returns a constrained efficient assignment.

The proof is not hard to sketch. At the end of Step 0, the MDA process, however specified, yields a stable assignment $\mu^{0}$. At every step $t \geq 1$ thereafter, if $\mu^{t}$ is not constrained efficient, we know from Proposition 3 that it must admit a PSIC. Moreover, the shortest such cycle will Pareto improve on $\mu^{t}$ while preserving stability. Until we reach a constrained efficient assignment, the algorithm will continue finding improvements which preserve stability. Since there are only finitely many improvements possible, we will end up with a constrained efficient assignment.15

The Floyd-Warshall algorithm ${ }^{16}$ is a computationally efficient way to find a shortest cycle in a directed graph. Each such cycle improves the temporary assignment for at

\footnotetext{
${ }^{15}$ The particular outcome of the PSIC algorithm will depend on the selections made in the MDA process in Step 0, and the specification of the cycle search in later steps. Remark 7 gives a characterization of substitutable priority structures for which PSIC returns an efficient assignment for all preference profiles.

${ }^{16}$ See Cormen, Leiserson, Rivest, and Stein (2001) for an exposition.
} 
least two students. There are $|X|$ schools, so each student can improve at most $|X|-1$ times, and therefore the total number of possible improvements is at most $|N|(|X|-1)$. Finally, the number of potential edges in the graph is at most $|N|(|N|-1)$, so the construction of the directed graph involves checking at most $|N|(|N|-1)$ pairs. Thus, PSIC is a polynomial time algorithm.

\subsection{Admissions by a committee}

Our second class of applications features highly flexible and multivalued admission rules which can be observed in real-life contexts ranging from undergraduate admissions at the University of Cambridge to course allocation schemes in various Japanese universities. Deferring the descriptions of these specific contexts to the Appendix, we introduce below what we call admissions by a committee.

Suppose an admissions committee consists of multiple referees where each referee has her own strict preference ranking (i.e., a linear order) over the set of students. When assessing a set of applications, referees can take turns to admit applicants according to their own ranking. For example, whenever they have time, they can access the admissions files and make selections. Selected students are put aside, and when a referee revisits the pool later, she will make selections from the pool of remaining applicants. Since the order in which the referees will make such choices is not fixed, there are many possible admissible sets ${ }^{17}$ Is this admission procedure substitutable? If all schools have admission rules of this sort, how can we find a constrained efficient assignment? To put it more formally, say a school has $q$ seats to fill. Denoting by $H$ its set of referees, every function $\pi:\{1, \ldots, q\} \rightarrow H$ leads to an associated admission rule $\mathcal{A}^{\pi}$ which selects students by sequentially appealing to referees in $H$ in the following fashion:

For any given $S \subseteq N$ with $|S|>q$,

- let $i_{1}$ be the highest ranked student in $S$ according to $\pi(1)$, and

\footnotetext{
${ }^{17}$ Alternatively, we can think of a single decision maker who can appeal to multiple criteria each of which is expressed as a ranking of students. Our formulation allows this decision maker to be flexible regarding the order in which she will appeal to these criteria when choosing candidates. This flexibility would be useful from an efficiency perspective if different orders would have different welfare consequences. While it might be difficult to know ex ante which orders would perform better, our PSIC process will be a practical way to explore such efficiency gains.
} 
- for each $k=2, \ldots, q$, let $i_{k}$ be the highest ranked student in $S \backslash\left\{i_{1}, \ldots, i_{k-1}\right\}$ according to $\pi(k)$,

and $\mathcal{A}^{\pi}(S)=\left\{i_{1}, \ldots, i_{q}\right\}$.

Each function $\pi:\{1, \ldots, q\} \rightarrow H$ specifies the sequence in which the school appeals to its referees. For example if $\pi$ is a constant function, a single referee will be deciding who is admitted. Kominers and Sönmez (2016) introduce these rules in the context of slot-specific priorities: (1) each seat has its own priority ranking, and (2) a precedence order over slots determines in which order the slots are filled. Since $\mathcal{A}^{\pi}$ is a rule of this sort, Kominers and Sönmez's results apply. In particular, $\mathcal{A}^{\pi}$ is a strict substitutable rule. The admissions committee, on the other hand, does not fix which priority rankings (referees) will be used nor in which order they will be used. Allowing for all possible ways in which the referees can take turns to select applicants means we have the multivalued admission rule

$$
\mathcal{A}^{H}(S)=\left\{\mathcal{A}^{\pi}(S) \mid \pi \in \Pi\right\}
$$

where $\Pi$ is the set of all functions $\pi:\{1, \ldots, q\} \rightarrow H$.

It is almost automatic to verify that a union of substitutable rules is substitutable. Hence we know $\mathcal{A}^{H}$ is indeed substitutable. If every school has a rule of this sort, the MDA process would find a stable assignment, but as before, arbitrary selections in the MDA process (or randomly fixing a particular $\pi$ ) can lead to the loss of constrained efficiency. The following result allows us to recover any such welfare loss.

Proposition 6 Suppose every school has an admission rule of the kind $\mathcal{A}^{H}$. A stable assignment is constrained efficient if and only if it does not admit a potentially-stable improvement cycle.

Hence, once again we can use PSIC to find a constrained efficient assignment.

Corollary 2 If every school has an admission rule of the kind $\mathcal{A}^{H}$, then the PSIC algorithm returns a constrained efficient assignment. 


\section{Conclusion}

Formulating substitutability for choice correspondences (i.e., multivalued choice rules) allowed us to study the problem of priority-based assignment in an environment which nests and goes beyond several classical settings. The first challenge in our general environment is that arbitrarily breaking ties in priorities might result in the DA process returning an unstable assignment. The MDA process, on the other hand, always returns a stable assignment. Next, the tension between stability and efficiency can be so extreme that all stable assignments might be inefficient even when all schools have identical priorities. Moreover, constrained efficiency (which is the obvious welfare objective) can be elusive, because there are too many ways to break ties to find a stable assignment, and it is not obvious which of those tie-breaking procedures lead to a constrained efficient outcome. We found an intuitive sufficient condition for an assignment to be constrained efficient, and showed that in two promising applications, this condition was also necessary, which in turn allowed us to design practical algorithms to find a constrained efficient assignment. Our analysis leaves open some important questions. Are there intuitive conditions on the priorities for which the tension between stability and efficiency disappears? What are some general conditions under which constrained efficiency is equivalent to lack of PSIC, so the PSIC algorithm is guaranteed to work? Further research can help elucidate and expand the use of our results.

\section{Acknowledgements}

We are grateful to Elizabeth Baldwin, Péter Biró, Lars Ehlers, Haluk Ergin, Paul Harrenstein, Onur Kesten, Philipp Kircher, Fuhito Kojima, Morimitsu Kurino, Al Roth, Zaifu Yang, seminar audiences at AMMA 2012, Cambridge, Edinburgh, Glasgow, Hitotsubashi University, Keio University, LBS, Lund, LSE, Nagoya University, Oxford, Royal Holloway, Washington University in St. Louis, York, and many other friends and colleagues for helpful discussions and advice. Kumano acknowledges financial support from the JSPS KAKENHI Grant Number 15K17019 and 17K13697. 


\section{Appendix}

\section{Admissions by a committee}

Here we describe two real-life contexts which motivate the specific class of rules we studied in Section 5.2 .

The admissions procedures at the University of Cambridge have both decentralized and centralized stages. The actual process is highly complex, but for our purposes, they can be summarised as follows. For admission to an undergraduate program at Cambridge, prospective students can either apply to one of the 31 colleges, or specify their application as an "open application". A significant portion of these applications are eventually forwarded to a "pool". Files of these applicants are available for each college admissions officer to evaluate. These admissions officers can freely access this pool over the course of a fixed time frame, and can "fish out" files from the pool to make them offers. All those applicants "fished out of the pool" are admitted to the university, and they comprise a significant portion of all students admitted. For example, in 2017 Autumn, 1075 prospective students applied to study economics at the University of Cambridge. Of these, 126 received direct offers, 230 others were placed in the pool, and 57 candidates were fished out of that pool of 230 applicants. That is, $31 \%$ of the offers were made through the pool, using an admission rule very much like what we called admissions by a committee.

In Japan, each undergraduate student is affiliated with a specific department from the beginning of their studies. In various universities, during the first two years of their studies, students are required to take a number of liberal arts courses in addition to department specific courses. When the demand for a particular course exceeds the number of available places for that course, those students with higher grade point averages (GPA) are given priority. However, comparing students in different departments according to such a metric is often considered unreasonable. Therefore, when assigning a place, a minimum fairness requirement is to make sure that the student who is assigned the seat has highest GPA among those students who demand a place and is from the same department. Since there are no requirements as to how places in a course should be distributed across different departments, there are many different admissible subsets. Interpreting GPA ranking in each department as the preference ranking of a 
distinct referee, the above course allocation scheme can also be seen as an example of admissions by a committee.

\section{Proof of Proposition 1}

$(\Rightarrow)$ This part is immediate since the conditions for pairwise stability are a special case of the conditions for group stability.

$(\Leftarrow)$ Denote $U=\mu^{-1}(x)$. If $\mu$ is pairwise stable, then it must be that for any $\ell$ with $x P_{\ell} \mu(\ell)$, we have $U \in \mathcal{A}_{x}(U \cup\{\ell\})$. Since students' preferences over schools are strict, we can write this in a seemingly stronger way: for any $\ell$ with $x R_{\ell} \mu(\ell)$, we have $U \in \mathcal{A}_{x}(U \cup\{\ell\})$. Given any $S \subseteq N$, if $x R_{i} \mu(i)$ for all $i \in S$, then we would like to show that $U \in \mathcal{A}_{x}(U \cup S)$. In order to conclude via induction on $|S|$, it is sufficient to show that

$$
\left[U \in \mathcal{A}_{x}(U \cup S) \text { and } U \in \mathcal{A}_{x}(U \cup\{k\})\right] \quad \Rightarrow \quad U \in \mathcal{A}_{x}(U \cup S \cup\{k\}) \text {. }
$$

Now, suppose for a contradiction that $U \notin \mathcal{A}_{x}(U \cup S \cup\{k\})$. Then for any $T \in$ $\mathcal{A}_{x}(U \cup S \cup\{k\})$, we know that $T$ is chosen instead of $U$. On the other hand, $U$ is chosen from among $U \cup S$. Therefore consistency ${ }^{18}$ of $\mathcal{A}_{x}$ implies that $T$ cannot be a subset of $U \cup S$, i.e., $T \nsubseteq U \cup S$. Since $T \subseteq U \cup S \cup\{k\}$, we conclude that $k \in T$. On the other hand, $U \in \mathcal{A}_{x}(U \cup\{k\})$ implies that $\{k\} \in \mathcal{R}_{x}(U \cup\{k\})$, which implies, due

\footnotetext{
${ }^{18}$ It is worth noting that the consistency property we assume for all of our admission rules is critical in establishing the equivalence between pairwise stability and group stability. For example, the rule defined as

$$
\begin{aligned}
\mathcal{A}_{x}(\{1,2,3,4\}) & =\{1,2\},\{3,4\} \\
\mathcal{A}_{x}(\{1,2,3\}) & =\{1,2\},\{2,3\} \\
\mathcal{A}_{x}(\{1,2,4\}) & =\{1,2\},\{2,4\} \\
\mathcal{A}_{x}(\{1,3,4\}) & =\{1,3\},\{3,4\} \\
\mathcal{A}_{x}(\{2,3,4\}) & =\{2,4\},\{3,4\}
\end{aligned}
$$

is substitutable. When the set of applicants is $\{1,2,4\}$, the sets $\{1,2\}$ and $\{2,4\}$ appear to be of equal priority, but when $\{1,2,3,4\}$ is the set of applicants, $\{1,2\}$ appears to have strictly higher priority than $\{2,4\}$. Hence $\mathcal{A}_{x}$ fails to satisfy consistency. Now, the assignment $\nu=(11,2 x, 33,4 x)$ is pairwise stable, because $\{2,4\}$ is an admissible set from both $\{1,2,4\}$ and $\{2,3,4\}$. On the other hand, $\nu$ is not group stable, because $\{2,4\}$ is not an admissible set from $\{1,2,3,4\}$.
} 
to substitutability, $\{k\} \subseteq(U \cup S \cup\{k\}) \backslash T$ for some $T \in \mathcal{A}_{x}(U \cup S \cup\{k\})$. But then $k \notin T$ yielding the desired contradiction.

\section{Proof of Proposition 2}

$A_{x}^{t}$ is the set of students who have applied to school $x$ in some round $k \leq t$. Hence

$$
A_{x}^{1} \subseteq A_{x}^{2} \subseteq \cdots
$$

The process requires that those students rejected in rounds $k \leq t-1$ would still be rejected if they were considered to be among the applicants in round $t$. This can be

ensured thanks to $\mathcal{A}_{x}$ being rejection monotonic, because $Z_{x}^{t-1}=A_{x}^{t-1} \backslash S_{x}^{\prime}$ for some $S_{x}^{\prime} \in \mathcal{A}_{x}\left(A_{x}^{t-1}\right)$ and $A_{x}^{t-1} \subseteq A_{x}^{t}$ together imply that there exists $Z_{x}^{t}=A_{x}^{t} \backslash S_{x}^{\prime \prime}$ such that $Z_{x}^{t} \supseteq Z_{x}^{t-1}$ for some $S_{x}^{\prime \prime} \in \mathcal{A}_{x}\left(A_{x}^{t}\right)$.

In order to see that the process indeed ends, note that at any round if a student is not matched, then she applies to her next favorite school in the following round. Therefore, she either exhausts all her acceptable schools by going down all the way to the end of her preference list, or ends up being matched with some school.

Suppose that the process ends at round $m$, and $\mu$ is the assignment obtained at the end. Students only apply to schools they find acceptable, so a student $i$ would only be matched with a school $x$ where $x R_{i} i$. Therefore $\mu$ is individually rational. Secondly, since $A_{x}^{m} \backslash \mu^{-1}(x)=Z_{x}^{m} \in \mathcal{R}_{x}\left(A_{x}^{m}\right)$, we have

$$
\mu^{-1}(x) \in \mathcal{A}_{x}\left(A_{x}^{m}\right) .
$$

Those who weakly prefer $x$ to their match under $\mu$ are either matched with $x$, or have applied to $x$ at some round of the process. Thus, $A_{x}^{m}=\left\{i \mid x R_{i} \mu(i)\right\}$. Now, since $\mu^{-1}(x) \in \mathcal{A}_{x}\left(\left\{i \mid x R_{i} \mu(i)\right\}\right), \mathcal{A}_{x}$ being acceptance monotonic implies $\mu^{-1}(x) \in \mathcal{A}_{x}(S)$ for any $S$ such that $\mu^{-1}(x) \subseteq S \subseteq\left\{i \mid x R_{i} \mu(i)\right\}$. Hence $\mu$ is stable.

\section{Proof of Proposition 3}

We will now show that if both $\mu$ and $\nu$ are stable, and if $\nu$ Pareto dominates $\mu$, then $\mu$ must admit a PSIC. From this, it will follow that if $\mu$ does not admit a PSIC, then it must be constrained efficient. 
Let $N^{\prime}=\{i \in N \mid \mu(i) \neq \nu(i)\}$ and $X^{\prime}=\left\{\nu(i) \mid i \in N^{\prime}\right\}$. For any $i \in N^{\prime}$, we know by the reshuffling lemma that $\mu(i) \in X^{\prime}$. Let $i \in N^{\prime}$, and $\mu(i)=x$. Denote

$$
D_{x}^{\mu}=\left\{j \in N \mid x P_{j} \mu(j)\right\}, \quad D_{x}^{\prime}=\left\{j \in N^{\prime} \mid x P_{j} \mu(j)\right\}, \quad D_{x}^{\prime \prime}=\left\{j \in N \backslash N^{\prime} \mid x P_{j} \mu(j)\right\}
$$

and set19

$$
\bar{D}_{x}=D_{x}^{\prime} \sqcup D_{x}^{\prime \prime} \sqcup \mu^{-1}(x)=D_{x}^{\mu} \sqcup \mu^{-1}(x) .
$$

The fact that $\mu$ is stable implies that

$$
\mu^{-1}(x) \in \mathcal{A}_{x}\left(\bar{D}_{x}\right)
$$

Moreover, stability of $\nu$ implies that

$$
D_{x}^{\prime \prime} \subseteq T^{\prime \prime} \text { for some } T^{\prime \prime} \in \mathcal{R}_{x}\left(D_{x}^{\nu} \sqcup \nu^{-1}(x)\right) \text {. }
$$

$\nu$ Pareto dominates $\mu$, so those who desire $x$ at $\nu$, desire $x$ at $\mu$ as well. Therefore $D_{x}^{\nu}=\left\{j \in N \mid x P_{j} \nu(j)\right\} \subseteq D_{x}^{\mu}$. Moreover, if $j \in \nu^{-1}(x)$, then either $j \in \mu^{-1}(x)$ or $j \in D_{x}^{\prime}$. And finally, since $\mu(i)=x$ and $i \in N^{\prime}$, we know that $i \notin \nu^{-1}(x)$, and $\nu(i) P_{i} x$. Therefore $i \notin D_{x}^{\nu}$. Thus

$$
D_{x}^{\nu} \sqcup \nu^{-1}(x) \subseteq D_{x}^{\mu} \cup \nu^{-1}(x) \subseteq \bar{D}_{x} \backslash\{i\}
$$

Now we conclude by using $(\star),(\star \star)$, and substitutability that

$$
D_{x}^{\prime \prime} \subseteq T^{\prime} \text { for some } T^{\prime} \in \mathcal{R}_{x}\left(\bar{D}_{x} \backslash\{i\}\right) .
$$

Denoting

$$
S^{\prime}=\left(\bar{D}_{x} \backslash\{i\}\right) \backslash T^{\prime},
$$

we have

$$
S^{\prime} \in \mathcal{A}_{x}\left(\bar{D}_{x} \backslash\{i\}\right) \quad \text { and } \quad S^{\prime} \cap D_{x}^{\prime \prime}=\varnothing
$$

Note that

$$
\bar{D}_{x} \backslash\{i\}=D_{x}^{\prime} \sqcup D_{x}^{\prime \prime} \sqcup\left[\mu^{-1}(x) \backslash\{i\}\right],
$$

and $\left|\mu^{-1}(x) \backslash\{i\}\right| \leq q_{x}-1$. Since $\mathcal{A}$ is acceptant, and $\left|\bar{D}_{x} \backslash\{i\}\right| \geq q_{x}$, we must have $\left|S^{\prime}\right| \geq q_{x}$. Because of $\left|\mu^{-1}(x) \backslash\{i\}\right| \leq q_{x}-1$ and that $S^{\prime} \cap D_{x}^{\prime \prime}=\varnothing$, we have

$$
S^{\prime} \cap D_{x}^{\prime} \neq \varnothing
$$

\footnotetext{
${ }^{19}$ We use $\sqcup$ to denote "disjoint union" throughout the Appendix.
} 
Hence, there exists $i^{\prime} \in D_{x}^{\prime}$ such that $\left\{i^{\prime}\right\} \cup\left[\mu_{x}^{-1} \backslash\{i\}\right] \in \mathcal{A}_{x}\left(\bar{D}_{x} \backslash\{i\}\right)$, i.e.,

$$
i^{\prime} \in E_{i}^{\mu} \text {. }
$$

Now construct a directed graph with $N^{\prime}$ being its set of vertices. For any $i \in N^{\prime}$, the above argument shows that there is $i^{\prime} \in N^{\prime}$ such that $i^{\prime} \in E_{i}^{\mu}$, so draw an edge $i^{\prime} \rightarrow i$. Since this is a finite graph with every vertex having an incoming edge, there must be a cycle. By construction, this is a PSIC.

\section{Proof of Proposition 4}

Lemma 1 If an admission rule satisfies $A M$ and $R M$ for every pair of sets $S \subseteq T$ with $|T \backslash S|=1$, then it is substitutable.

Proof of Lemma 1. We will verify that AM and RM hold for every pair of sets $S \subseteq T$ by doing strong induction on $k=|T \backslash S|$. We are given that the conditions hold for $k=1$. Now assuming they hold for $k \leq m-1$, we will verify them for $k=m$. Let $|T \backslash S|=m$.

(AM) Given $T^{\prime} \in \mathcal{A}(T)$, pick some $t \in T \backslash T^{\prime}$, and set $\tilde{T}=T \backslash\{t\}$. By the induction hypothesis, $T^{\prime} \cap \tilde{T} \subseteq T^{\prime \prime}$ for some $T^{\prime \prime} \in \mathcal{A}(\tilde{T})$. Since $T^{\prime} \cap \tilde{T}=T^{\prime}$ and $\mathcal{A}$ is acceptant, $T^{\prime}=T^{\prime \prime}$ and $T^{\prime} \in \mathcal{A}(\tilde{T})$.

If $t \notin S$, then $S \subseteq \tilde{T}$. Since $|\tilde{T} \backslash S|=m-1$, by the induction hypothesis $T^{\prime} \cap S \subseteq S^{\prime}$ for some $S^{\prime} \in \mathcal{A}(S)$.

If, on the other hand, $t \in S$ for all $t \in T \backslash T^{\prime}$, then let $\hat{T}=T \backslash\{\hat{t}\}$ for some $\hat{t} \notin S$. Such $\hat{t}$ is necessarily in $T^{\prime}$. Again, by the induction hypothesis, $T^{\prime} \backslash\{\hat{t}\}=T^{\prime} \cap \hat{T} \subseteq T^{\prime \prime}$ for some $T^{\prime \prime} \in \mathcal{A}(\hat{T})$.

$S \subseteq \hat{T}$, because $\hat{t} \notin S$. Using the induction hypothesis

$$
T^{\prime} \cap S \subseteq\left(T^{\prime \prime} \sqcup\{\hat{t}\}\right) \cap S=T^{\prime \prime} \cap S \subseteq S^{\prime \prime} \quad \text { for some } \quad S^{\prime \prime} \in \mathcal{A}(S) .
$$

$\underline{(\mathrm{RM})}$ Given $S^{\prime} \in \mathcal{A}(S)$, let $t \in T \backslash S$ and $\tilde{T}=T \backslash\{t\}$. Then $S \subseteq \tilde{T}$ and $|\tilde{T} \backslash S|=k-1$, so by the induction hypothesis, there must exist $\tilde{T}^{\prime} \in \mathcal{A}(\tilde{T})$ such that $S \backslash S^{\prime} \subseteq \tilde{T} \backslash \tilde{T}^{\prime}$.

$\tilde{T} \subseteq T$ and $|T \backslash \tilde{T}|=1$, so again by the induction hypothesis, there exists $T^{\prime} \in \mathcal{A}(T)$ such that $\tilde{T} \backslash \tilde{T}^{\prime} \subseteq T \backslash T^{\prime}$.

Putting together the two derived inclusions, we conclude $S \backslash S^{\prime} \subseteq \tilde{T} \backslash \tilde{T}^{\prime} \subseteq T \backslash T^{\prime}$, where $T^{\prime} \in \mathcal{A}(T)$. 


\section{Computing an EDCR admission rule}

Given an EDCR admission rule, we now describe a procedure to compute the set of admissible subsets of $S$ for every $S \subseteq N$. Afterwards, we will use this procedure to help prove Proposition 4.

The distribution of types in $S$ is the vector $\boldsymbol{v}(S)=\left(v_{\tau}\right)_{\tau \in \mathcal{T}}$, where $v_{\tau}$ is the number of type- $\tau$ students in $S$, i.e., $v_{\tau}=\left|S_{\tau}\right|$. A set $S^{\prime} \subseteq S$ is a set of top- $t$ students in $S$ with respect to $\succsim^{e x o}$ if $\left|S^{\prime}\right|=t$ and $s^{\prime} \succsim^{e x o} s$ for all $s^{\prime} \in S^{\prime}$ and $s \in S \backslash S^{\prime}$. A student is called a $t+1$ st student in $S$ with respect to $\succsim^{e x o}$ if he belongs to a set of top- $(t+1)$ students in $S$, but does not belong to any set of top- $t$ students in $S$ with respect to $\succsim^{e x o} . \quad \boldsymbol{a}=\left(a_{\tau}\right)_{\tau \in \mathcal{T}}$ is called an admissible distribution from $S$ if there exists $S^{\prime} \in \mathcal{A}_{x}(S)$ such that the distribution of types in $S^{\prime}$ is $\boldsymbol{a}$. We will denote by $\boldsymbol{\alpha}(S)$ the set of admissible distributions from $S$. Hence the set of admissible subsets $\mathcal{A}_{x}(S)$ of $S$ can be expressed as

$$
\left\{\begin{array}{l|l}
S^{\prime} \subseteq S & \begin{array}{l}
\boldsymbol{v}\left(S^{\prime}\right)=\boldsymbol{a} \text { for some } \boldsymbol{a} \in \boldsymbol{\alpha}(S), \text { and } \\
S_{\tau}^{\prime} \text { is a set of top- } a_{\tau} \text { students in } S_{\tau} \text { with respect to } \succsim^{e x o}
\end{array}
\end{array}\right\} .
$$

In order to give a more explicit description of the admission rule $\mathcal{A}_{x}$, let us construct, in detail, the set $\boldsymbol{\alpha}(S)$ of admissible distributions.

Step 0: Fill as many seats as possible with the types intended for those seats, i.e., set

$$
a_{\tau}^{0}= \begin{cases}\left|S_{\tau}\right| & \text { for all } \tau \text { such that }\left|S_{\tau}\right| \leq q_{x}^{\tau} \\ q_{x}^{\tau} & \text { for all } \tau \text { such that }\left|S_{\tau}\right|>q_{x}^{\tau}\end{cases}
$$

and define the number of surplus seats at the end of step 0 as $e_{1}=q_{x}-\sum_{\tau} a_{\tau}^{0}$ which is non-negative. If $e_{1}=0$, then there is a unique admissible distribution: $\boldsymbol{q}_{x}$. Otherwise, $e_{1}>0$, and we determine each admissible distribution by following the steps below for $k \geq 0$ :

Step $k$ for $k \geq 1$ : If the number of types of remaining applicants not yet assigned at the end of step $k-1$ is greater than or equal to $e_{k}$, i.e., if $\left|\left\{\tau:\left|S_{\tau}\right|>a_{\tau}^{k-1}\right\}\right| \geq e_{k}$, then assign the $e_{k}$ surplus seats to remaining types according to the priority order $\succsim^{e x o}$, and with at most one surplus seat to each type. Formally speaking, for each $\tau$ with $\left|S_{\tau}\right|>a_{\tau}^{k-1}$, let $i_{\tau}$ be an $a_{\tau}^{k}$ th student in $S_{\tau}$ with respect to $\succsim^{e x o}$. For every $U$ which is 
a set of top- $e_{k}$ students in $\left\{i_{\tau}:\left|S_{\tau}\right|>a_{\tau}^{k-1}\right\}$ with respect to $\succsim^{\text {exo }}$, set

$$
a_{\tau}^{U}= \begin{cases}a_{\tau}^{k-1}+1 & \text { for all } \tau \text { such that } i_{\tau} \in U \\ a_{\tau}^{k-1} & \text { otherwise }\end{cases}
$$

to obtain an admissible distribution $\boldsymbol{a}^{U}=\left(a_{\tau}^{U}\right)_{\tau \in \mathcal{T}}$. Thus we conclude

$$
\boldsymbol{\alpha}(S)=\left\{\boldsymbol{a}^{U}: U \text { is a set of top- } e_{k} \text { students in }\left\{i_{\tau}:\left|S_{\tau}\right|>a_{\tau}^{k-1}\right\}\right\}
$$

If, however, $\left|\left\{\tau:\left|S_{\tau}\right|>a_{\tau}^{k-1}\right\}\right|<e_{k}$, then set

$$
a_{\tau}^{k}= \begin{cases}a_{\tau}^{k-1} & \text { for all } \tau \text { such that }\left|S_{\tau}\right| \leq a_{\tau}^{k-1} \\ a_{\tau}^{k-1}+1 & \text { for all } \tau \text { such that }\left|S_{\tau}\right|>a_{\tau}^{k-1}\end{cases}
$$

and $e_{k+1}=e_{k}-\left|\left\{\tau:\left|S_{\tau}\right|>a_{\tau}^{k-1}\right\}\right|=q_{x}-\sum_{\tau} a_{\tau}^{k}>0$, and go to Step $k+1$.

Proof of Proposition 4. Thanks to Lemma 1, it is sufficient to verify AM and RM for sets $S$ and $S \cup\{t\}$, where $|S| \geq q_{x}$. When, $|S| \leq q_{x}$, both conditions are easily verified. Hence, we will assume below that $|S| \geq q_{x}+1$.

Proof of $A M$. Let $T^{\prime} \in \mathcal{A}(S \cup\{t\})$. Consider the specific steps of the procedure which results in $T^{\prime}$ being chosen from $S \cup\{t\}$. Say it took $n \geq 1$ steps for this procedure to end.

If $t \notin T^{\prime}$, then $t$ stayed in the pool of applicants until the end of the procedure, and was not chosen. That means, in Step 0, exactly $q_{x}^{\tau}$ students of type $\tau=\tau(t)$ were chosen, and at steps 2 to $n-1$, exactly one student of type $\tau(t)$ was chosen. Moreover all of these students are of weakly higher priority than $t$ according to $\succsim^{e x o}$. Finally, in step $n$, all chosen students are of weakly higher priority than $t$ according to $\succsim^{e x o}$. The same steps of this procedure (with exactly the same choices at each step) can be repeated when the set of applicants is $S$. Hence $T^{\prime} \cap S=T^{\prime} \in \mathcal{A}(S)$.

If, on the other hand, $t \in T^{\prime}$, then say she was chosen in step $p$ of the procedure. If $p=n$, that is, if $t$ was chosen in the very last step of the procedure, the first $n-1$ steps of the procedure can be repeated (with exactly the same choices at each step) when the set of applicants is $S$. Since $|S \cup\{t\}| \geq q_{x}+2$, there were at least two students remaining in step $n$. When $t$ is removed from the original set of applicants, each student $s$ who had been selected in step $n$ remains in step $n$, and is still selected because $s \succsim^{\text {exo }} s^{\prime}$ for every remaining $s^{\prime}$ with $\tau\left(s^{\prime}\right)=\tau(s)$. Thus $T^{\prime} \cap S=T^{\prime} \backslash\{t\} \subset S^{\prime}$ for some $S^{\prime} \in \mathcal{A}(S)$. 
Otherwise, $t \in T^{\prime}$, and she was chosen in step $p<n$ of the procedure. Say the last chosen student of type $\tau(t)$ was chosen in step $r$. So $p \leq r \leq n$. When we apply the procedure to $S$ to choose an admissible set, the first $p-1$ steps can be replicated from the original procedure we had fixed for choosing from $S \cup\{t\}$. The rest of the new procedure will continue as follows: for each step $j=p, \ldots, r-1$, the type- $\tau(t)$ student who was chosen in step $j+1$ of the original procedure is chosen in step $j$ of the new procedure. As for students of other types, those who were chosen in steps $j=1, \ldots, n$ continue to be chosen in the same steps of the new procedure. In the new procedure, there is one more remaining seat in step $n$. Independent of how that new surplus seat is assigned, we have made sure that every student who was chosen from $S \cup\{t\}$ continues to be chosen from $S$; which completes the proof of AM.

Proof of $R M$. Let $S^{\prime} \in \mathcal{A}(S)$. We need to show that there exists $T^{\prime} \in \mathcal{A}(S \cup\{t\})$ such that $S \backslash S^{\prime} \subseteq(S \cup\{t\}) \backslash T^{\prime}$. Given the specific procedure which resulted in $S^{\prime}$ being chosen from $S$, we will argue that there is a procedure of choice from $S \cup\{t\}$ so that no student in $S \backslash S^{\prime}$ is chosen. Note that all students in $S \backslash S^{\prime}$ are among the remaining students in step $n$ of the original procedure. In particular, for every $s \in S \backslash S^{\prime}$, there exist $q_{x}^{\tau}(s)+n-1$ students of type $\tau(s)$ who have weakly higher $\succsim^{\text {exo }}$ priority than $s$. Since all such students are also present in the new procedure, student $s$ can still be rejected in the first $n$ steps of the new procedure. Having the extra applicant $t$ will decrease the number of steps the procedure will take, and therefore, the new procedure will finish in at most $n$ steps. Thus, the new procedure can be implemented so that all those rejected from $S$ in the original procedure are rejected in the new procedure when choosing from $S \cup\{t\}$.

\section{Proof of Proposition 5}

It follows from Propositions 3 and 4 that if a stable assignment does not admit a PSIC, then it is constrained efficient. For the other direction, we are first going to establish that if a PSIC "cannot be shortened", then it preserves stability. It, then, suffices to observe that if $\mu$ is stable and admits a PSIC, then there must be a shortest of all such PSICs, and that shortest PSIC leads to an assignment which Pareto dominates $\mu$ while preserving stability. 
Definition 1 A PSIC

$$
i_{0} \rightarrow i_{1} \rightarrow \cdots \rightarrow i_{n} \rightarrow i_{n+1}=i_{0}
$$

is called minimal if there do not exist $s<t$ such that

$$
i_{0} \rightarrow i_{1} \rightarrow \cdots \rightarrow i_{s} \rightarrow i_{t+1} \rightarrow \cdots \rightarrow i_{n} \rightarrow i_{n+1}=i_{0}
$$

is a PSIC.

Lemma 2 If the priority structure satisfies EDCR, then in each minimal PSIC

(a) those students who move to the same school are of distinct types,

(b) those students who leave the same school are of distinct types.

Proof of Lemma 2. Given stable $\mu$, let $\nu$ be the assignment obtained from $\mu$ by carrying out a minimal PSIC. Label those students matched with school $x$ under $\nu$ as $i_{k^{1}}, \ldots, i_{k^{m}}$, where PSIC looks like

$$
i_{0} \rightarrow i_{1} \rightarrow \cdots \rightarrow i_{k^{t}} \rightarrow i_{k^{t}+1} \rightarrow \cdots \rightarrow i_{n} \rightarrow i_{n+1}=i_{0}
$$

Hence $\mu\left(i_{k^{t}+1}\right)=\nu\left(i_{k^{t}}\right)=x$ for all $t \in\{1, \cdots, m\}$.

Proof of Part (a) We need to show that $\tau\left(i_{k^{t}}\right) \neq \tau\left(i_{k^{u}}\right)$ for every $t, u \in\{1, \ldots, m\}$ and $t \neq u$. Assume otherwise, and thus $\tau=\tau\left(i_{k^{t}}\right)=\tau\left(i_{k^{u}}\right)$ for some $t$ and $u \neq t$.

Since $i_{k^{t}} \in E_{i_{k^{t}+1}}^{\mu}$ and $i_{k^{u}} \in \mathcal{E}_{i_{k^{t}+1}}^{\mu}$,

$$
\left[\mu^{-1}(x) \backslash\left\{i_{k^{t}+1}\right\}\right] \sqcup\left\{i_{k^{t}}\right\} \quad \succsim \quad\left[\mu^{-1}(x) \backslash\left\{i_{k^{t}+1}\right\}\right] \sqcup\left\{i_{k^{u}}\right\} .
$$

In what follows, for notational convenience we will let write $\operatorname{ed}(S) \operatorname{instead}$ of $\operatorname{ed}\left(\boldsymbol{q}_{x}, S\right)$. Since $i_{k^{t}}$ and $i_{k^{u}}$ are of the same type, we have

$$
\operatorname{ed}\left(\left[\mu^{-1}(x) \backslash\left\{i_{k^{t}+1}\right\}\right] \sqcup\left\{i_{k^{t}}\right\}\right)=\operatorname{ed}\left(\left[\mu^{-1}(x) \backslash\left\{i_{k^{t}+1}\right\}\right] \sqcup\left\{i_{k^{u}}\right\}\right),
$$

which implies $i_{k^{t}} \succsim_{x}^{e x o} i_{k^{u}}$.

Using a symmetric argument, we must have $i_{k^{u}} \succsim_{x}^{e x o} i_{k^{t}}$, and therefore $i_{k^{t}} \sim_{x}^{\text {exo }} i_{k^{u}}$. Hence $i_{k^{u}} \in E_{k^{t}+1}^{\mu}$, allowing us to construct a shorter PSIC, which contradicts with the minimality of the PSIC we started with. 
Proof of Part (b) Suppose, for a contradiction, that $\tau\left(i_{k^{t}+1}\right)=\tau\left(i_{k^{s}+1}\right)$ for some $t \neq s$. Then the sets $\mu^{-1}(x) \backslash\left\{i_{k^{t}+1}\right\}$ and $\mu^{-1}(x) \backslash\left\{i_{k^{s}+1}\right\}$ have the same distributions of types. Therefore

$$
\operatorname{ed}\left(\left[\mu^{-1}(x) \backslash\left\{i_{k^{t}+1}\right\}\right] \cup\left\{i_{k^{t}}\right\}\right)=\operatorname{ed}\left(\left[\mu^{-1}(x) \backslash\left\{i_{k^{s}+1}\right\}\right] \cup\left\{i_{k^{t}}\right\}\right)
$$

and

$$
\operatorname{ed}\left(\left[\mu^{-1}(x) \backslash\left\{i_{k^{t}+1}\right\}\right] \cup\left\{i_{k^{s}}\right\}\right)=\operatorname{ed}\left(\left[\mu^{-1}(x) \backslash\left\{i_{k^{s}+1}\right\}\right] \cup\left\{i_{k^{s}}\right\}\right) .
$$

Next, note that $i_{k^{t}} \in E_{i_{k^{t}+1}}^{\mu}$ and $i_{k^{s}} \in \mathcal{E}_{i_{k^{t}+1}}^{\mu}$ together imply

$$
\operatorname{ed}\left(\left[\mu^{-1}(x) \backslash\left\{i_{k^{t}+1}\right\}\right] \cup\left\{i_{k^{t}}\right\}\right) \leq \operatorname{ed}\left(\left[\mu^{-1}(x) \backslash\left\{i_{k^{t}+1}\right\}\right] \cup\left\{i_{k^{s}}\right\}\right) .
$$

If $(\star)$ is a strict inequality, substituting $(*)$ and $(* *)$ into $(\star)$ yields

$$
\operatorname{ed}\left(\left[\mu^{-1}(x) \backslash\left\{i_{k^{s}+1}\right\}\right] \cup\left\{i_{k^{t}}\right\}\right)<\operatorname{ed}\left(\left[\mu^{-1}(x) \backslash\left\{i_{k^{s}+1}\right\}\right] \cup\left\{i_{k^{s}}\right\}\right),
$$

which, combined with $i_{k^{t}} \in \mathcal{E}_{i_{k^{t}+1}}^{\mu}=\mathcal{E}_{i_{k^{s}+1}}^{\mu}$, implies $i_{k^{s}+1} \notin \mathcal{E}_{i_{k^{s}+1}}^{\mu}$, a contradiction.

Thus, $(\star)$ is an equality, and therefore we must have $i_{k^{t}} \succsim_{x}^{\text {exo }} i_{k^{s}}$. Reversing the roles of $t$ and $s$ above, and repeating the arguments, we then conclude $i_{k^{s}} \succsim_{x}^{\text {exo }} i_{k^{t}}$, and therefore $i_{k^{t}} \sim_{x}^{e x o} i_{k^{s}}$.

Now, the fact that $(\star)$ is an equality and $i_{k^{t}} \sim_{x}^{e x o} i_{k^{s}}$ imply $i_{k^{s}} \in E_{i_{k^{t}+1}}^{\mu}$, which leads to a shorter PSIC than the one we started with; a contradiction.

Proof of Proposition 5. Assume, for a contradiction, that $\mu$ is constrained efficient but admits a PSIC. Let $\nu$ be the assignment obtained by carrying out the shortest PSIC $\mu$ admits. Since $\nu$ Pareto dominates $\mu$ which is constrained efficient to begin with, $\nu$ cannot be stable. EDCR admission rules are consistent, so stability (i.e., respecting priorities) is equivalent to pairwise stability, and therefore there exist $x \in X$ and $j \in N$ such that $x P_{j} \nu(j)$ and $\nu^{-1}(x) \notin \mathcal{A}_{x}\left(\nu^{-1}(x) \cup\{j\}\right)$.

Consider, now, the students involved in the above shortest PSIC and label those students matched with $x$ under $\nu$ as $i_{k^{1}}, \ldots, i_{k^{m}}$, where the PSIC looks like

$$
i_{0} \rightarrow i_{1} \rightarrow \cdots \rightarrow i_{k^{t}} \rightarrow i_{k^{t}+1} \rightarrow \cdots \rightarrow i_{n} \rightarrow i_{n+1}=i_{0} .
$$

Hence $\mu\left(i_{k^{t}+1}\right)=\nu\left(i_{k^{t}}\right)=x$ for all $t \in\{1, \cdots, m\}$.

Since $\nu^{-1}(x) \notin \mathcal{A}_{x}\left(\nu^{-1}(x) \cup\{j\}\right)$, there must exist $\ell \in \nu^{-1}(x)$ such that $\left(\nu^{-1}(x) \backslash\{\ell\}\right) \cup$ $\{j\} \succ_{x} \nu^{-1}(x)$. 
Setting $A=\left(\nu^{-1}(x) \backslash\{\ell\}\right) \cup\{j\}$, we must have $\operatorname{ed}\left(\boldsymbol{q}_{x}, A\right) \leq \operatorname{ed}\left(\boldsymbol{q}_{x}, \nu^{-1}(x)\right)$, where $j \succ_{x}^{e x o} \ell$ whenever equality holds.

Case 1: There are at least two types $t, s \neq t$ such that $\tau\left(i_{k^{t}}\right) \neq \tau\left(i_{k^{t}+1}\right)$ and $\tau\left(i_{k^{s}}\right) \neq$ $\tau\left(i_{k^{s}+1}\right)$.

Case 1.1: $\tau\left(i_{k^{t}}\right)=\tau\left(i_{k^{s}+1}\right)$ or $\tau\left(i_{k^{s}}\right)=\tau\left(i_{k^{t}+1}\right)$.

Without loss of generality, we assume that $\tau\left(i_{k^{t}}\right)=\tau\left(i_{k^{s}+1}\right)$. Then since $i_{k^{s}} \in E_{i_{k^{s}+1}}^{\mu}$ and $\mu$ is stable, we must have

$$
\begin{aligned}
\operatorname{ed}\left(\boldsymbol{q}_{x}, \mu^{-1}(x)\right) \leq \operatorname{ed}\left(\boldsymbol{q}_{x},\left[\mu^{-1}(x) \backslash\left\{i_{k^{s}+1}\right\}\right] \cup\left\{i_{k^{s}}\right\}\right) & \leq \operatorname{ed}\left(\boldsymbol{q}_{x},\left[\mu^{-1}(x) \backslash\left\{i_{k^{s}+1}\right\}\right] \cup\left\{i_{k^{t}}\right\}\right) \\
& =\operatorname{ed}\left(\boldsymbol{q}_{x}, \mu^{-1}(x)\right)
\end{aligned}
$$

so that

$$
i_{k^{s}} \succsim_{x}^{e x o} i_{k^{t}}
$$

If $i_{k^{s}} \sim_{x}^{e x o} i_{k^{t}}$, then $i_{k^{t}} \in E_{i_{k^{s}+1}}^{\mu}$, a contradiction to the cycle is minimal. Therefore, we have $i_{k^{s}} \succ_{x}^{e x o} i_{k^{t}}$. Together with $i_{k^{t}} \in E_{i_{k^{t}+1}}^{\mu}$, this implies that

$$
\operatorname{ed}\left(\boldsymbol{q}_{x},\left[\mu^{-1}(x) \backslash\left\{i_{k^{t}+1}\right\}\right] \cup\left\{i_{k^{t}}\right\}\right)<\operatorname{ed}\left(\boldsymbol{q}_{x},\left[\mu^{-1}(x) \backslash\left\{i_{k^{t}+1}\right\}\right] \cup\left\{i_{k^{s}}\right\}\right),
$$

thus $\tau\left(i_{k^{s}}\right) \neq \tau\left(i_{k^{t}+1}\right)$.

Since $\tau\left(i_{k^{t}}\right) \neq \tau\left(i_{k^{t}+1}\right)$ (from Part (b) of Lemma 22) and $\tau\left(i_{k^{s}}\right) \neq \tau\left(i_{k^{t}+1}\right)$, ed $\left(\boldsymbol{q}_{x},\left[\mu^{-1}(x) \backslash\left\{i_{k^{t}+1}\right\}\right] \cup\right.$ $\left.\left\{i_{k^{t}}\right\}\right)<\operatorname{ed}\left(\boldsymbol{q}_{x},\left[\mu^{-1}(x) \backslash\left\{i_{k^{t}+1}\right\}\right] \cup\left\{i_{k^{s}}\right\}\right)$ implies that

$$
\begin{aligned}
& \left(\left|\left(\mu^{-1}(x)\right)_{\tau\left(i_{k^{t}}\right)}\right|+1-q_{x}^{\tau\left(i_{k^{t}}\right)}\right)^{2}+\left(\left|\left(\mu^{-1}(x)\right)_{\tau\left(i_{k^{s}}\right)}\right|-q_{x}^{\tau\left(i_{k^{s}}\right)}\right)^{2}< \\
& \left(\left|\left(\mu^{-1}(x)\right)_{\tau\left(i_{k^{t}}\right)}\right|-q_{x}^{\tau\left(i_{k^{t}}\right)}\right)^{2}+\left(\left|\left(\mu^{-1}(x)\right)_{\tau\left(i_{k^{s}}\right)}\right|+1-q_{x}^{\tau\left(i_{k^{s}}\right)}\right)^{2} .
\end{aligned}
$$

Setting $a=\left|\left(\mu^{-1}(x)\right)_{\tau\left(i_{k} t\right.}\right|-q_{x}^{\tau\left(i_{k^{t}}\right)}$, we have $\left|\left(\mu^{-1}(x)\right)_{\tau\left(i_{k^{s}}\right)}\right|-q_{x}^{\tau\left(i_{k^{s}}\right)}>a$.

We also have ed $\left(\boldsymbol{q}_{x},\left[\mu^{-1}(x) \backslash\left\{i_{k^{s}+1}\right\}\right] \cup\left\{i_{k^{s}}\right\}\right)=\operatorname{ed}\left(\boldsymbol{q}_{x},\left[\mu^{-1}(x) \backslash\left\{i_{k^{s}+1}\right\}\right] \cup\left\{i_{k^{t}}\right\}\right)$, which implies that

$$
\begin{gathered}
\left(\left|\left(\mu^{-1}(x)\right)_{\tau\left(i_{k^{t}}\right)}\right|-1-q_{x}^{\tau\left(i_{k^{t}}\right)}\right)^{2}+\left(\left|\left(\mu^{-1}(x)\right)_{\tau\left(i_{k^{s}}\right)}\right|+1-q_{x}^{\tau\left(i_{k^{s}}\right)}\right)^{2}= \\
\left(\left|\left(\mu^{-1}(x)\right)_{\tau\left(i_{k^{t}}\right)}\right|-q_{x}^{\tau\left(i_{k^{t}}\right)}\right)^{2}+\left(\left|\left(\mu^{-1}(x)\right)_{\tau\left(i_{k^{s}}\right)}\right|-q_{x}^{\tau\left(i_{k^{s}}\right)}\right)^{2}
\end{gathered}
$$

which implies that $\left|\left(\mu^{-1}(x)\right)_{\tau\left(i_{k^{s}}\right)}\right|-q_{x}^{\tau\left(i_{k} s\right)}=a-1$, a contradiction. 
Case 1.2: $\tau\left(i_{k^{t}}\right) \neq \tau\left(i_{k^{s}+1}\right)$ and $\tau\left(i_{k^{s}}\right) \neq \tau\left(i_{k^{t}+1}\right)$.

We claim that in this case we can find a shorter cycle. Since $\mu$ is stable, we must have $\left|\left(\mu^{-1}(x)\right)_{\tau\left(i_{k} t\right)}\right| \geq q_{x}^{\tau\left(i_{k^{t}}\right)}$ and $\left|\left(\mu^{-1}(x)\right)_{\tau\left(i_{k^{s}}\right)}\right| \geq q_{x}^{\tau\left(i_{k^{s}}\right)}$.

$i_{k^{t}} \in E_{i_{k^{t}+1}}^{\mu}$ and $i_{k^{s}} \in \mathcal{E}_{i_{k^{t}+1}}^{\mu}$ imply that

$$
\operatorname{ed}\left(\boldsymbol{q}_{x},\left[\mu^{-1}(x) \backslash\left\{i_{k^{t}+1}\right\}\right] \cup\left\{i_{k^{t}}\right\}\right) \leq \operatorname{ed}\left(\boldsymbol{q}_{x},\left[\mu^{-1}(x) \backslash\left\{i_{k^{t}+1}\right\}\right] \cup\left\{i_{k^{s}}\right\}\right) .
$$

Since $\tau\left(i_{k^{t}}\right) \neq \tau\left(i_{k^{t}+1}\right) \neq \tau\left(i_{k^{s}}\right)$,

$$
\begin{aligned}
& \left(\left|\left(\mu^{-1}(x)\right)_{\tau\left(i_{k^{t}}\right)}\right|+1-q_{x}^{\tau\left(i_{k^{t}}\right)}\right)^{2}+\left(\left|\left(\mu^{-1}(x)\right)_{\tau\left(i_{k^{s}}\right)}\right|-q_{x}^{\tau\left(i_{k^{s}}\right)}\right)^{2} \leq \\
& \left(\left|\left(\mu^{-1}(x)\right)_{\tau\left(i_{k^{t}}\right)}\right|-q_{x}^{\tau\left(i_{k^{t}}\right)}\right)^{2}+\left(\left|\left(\mu^{-1}(x)\right)_{\tau\left(i_{k^{s}}\right)}\right|+1-q_{x}^{\tau\left(i_{k^{s}}\right)}\right)^{2} .
\end{aligned}
$$

Similarly, $i_{k^{s}} \in E_{i_{k^{s}+1}}^{\mu}$ and $i_{k^{t}} \in \mathcal{E}_{i_{k^{s}+1}}^{\mu}$ imply that

$$
\operatorname{ed}\left(\boldsymbol{q}_{x},\left[\mu^{-1}(x) \backslash\left\{i_{k^{s}+1}\right\}\right] \cup\left\{i_{k^{s}}\right\}\right) \leq \operatorname{ed}\left(\boldsymbol{q}_{x},\left[\mu^{-1}(x) \backslash\left\{i_{k^{s}+1}\right\}\right] \cup\left\{i_{k^{t}}\right\}\right) .
$$

Since $\tau\left(i_{k^{s}}\right) \neq \tau\left(i_{k^{s}+1}\right) \neq \tau\left(i_{k^{t}}\right)$,

$$
\begin{aligned}
& \left(\left|\left(\mu^{-1}(x)\right)_{\tau\left(i_{k^{t}}\right)}\right|+1-q_{x}^{\tau\left(i_{k}\right)}\right)^{2}+\left(\left|\left(\mu^{-1}(x)\right)_{\tau\left(i_{k^{s}}\right)}\right|-q_{x}^{\tau\left(i_{k^{s}}\right)}\right)^{2} \geq \\
& \left(\left|\left(\mu^{-1}(x)\right)_{\tau\left(i_{k^{t}}\right)}\right|-q_{x}^{\tau\left(i_{k^{t}}\right)}\right)^{2}+\left(\left|\left(\mu^{-1}(x)\right)_{\tau\left(i_{k^{s}}\right)}\right|+1-q_{x}^{\tau\left(i_{k^{s}}\right)}\right)^{2} .
\end{aligned}
$$

Thus,

$$
\begin{gathered}
\left(\left|\left(\mu^{-1}(x)\right)_{\tau\left(i_{k^{t}}\right)}\right|+1-q_{x}^{\tau\left(i_{k}\right)}\right)^{2}+\left(\left|\left(\mu^{-1}(x)\right)_{\tau\left(i_{k} s\right)}\right|-q_{x}^{\tau\left(i_{k} s\right)}\right)^{2}= \\
\left(\left|\left(\mu^{-1}(x)\right)_{\tau\left(i_{k^{t}}\right)}\right|-q_{x}^{\tau\left(i_{k^{t}}\right)}\right)^{2}+\left(\left|\left(\mu^{-1}(x)\right)_{\tau\left(i_{k^{s}}\right)}\right|+1-q_{x}^{\tau\left(i_{k^{s}}\right)}\right)^{2},
\end{gathered}
$$

which implies that $i_{k^{t}} \sim_{x}^{e x o} i_{k^{s}}$. Hence $i_{k^{s}} \in E_{i_{k^{t}+1}}^{\mu}$, and we find a shorter cycle

$$
i_{0} \rightarrow i_{1} \rightarrow \cdots \rightarrow i_{k^{s}} \rightarrow i_{k^{t}+1} \rightarrow \cdots \rightarrow i_{n} \rightarrow i_{n+1}=i_{0}
$$

which contradicts that the original cycle is minimal.

Case 2: There exists a unique $t$ such that $\tau\left(i_{k^{t}}\right) \neq \tau\left(i_{k^{t}+1}\right)$; for all $s \neq t, \tau\left(i_{k^{s}}\right)=$ $\tau\left(i_{k^{s}+1}\right)$.

Suppose $\ell=i_{k^{t}}$. Note that

$$
\operatorname{ed}\left(\boldsymbol{q}_{x},\left[\mu^{-1}(x) \backslash\left\{i_{k^{t}+1}\right\}\right] \cup\left\{i_{k^{t}}\right\}\right)=\operatorname{ed}\left(\boldsymbol{q}_{x}, \nu^{-1}(x)\right) .
$$


Then since $i_{k^{t}} \in E_{i_{k^{t}+1}}^{\mu}$,

$$
\operatorname{ed}\left(\boldsymbol{q}_{x},\left[\mu^{-1}(x) \backslash\left\{i_{k^{t}+1}\right\}\right] \cup\left\{i_{k^{t}}\right\}\right) \leq \operatorname{ed}\left(\boldsymbol{q}_{x},\left[\mu^{-1}(x) \backslash\left\{i_{k^{t}+1}\right\}\right] \cup\{j\}\right) .
$$

If $\operatorname{ed}\left(\boldsymbol{q}_{x}, A\right)<\operatorname{ed}\left(\boldsymbol{q}_{x}, \nu^{-1}(x)\right)$ then

$$
\operatorname{ed}\left(\boldsymbol{q}_{x},\left[\mu^{-1}(x) \backslash\left\{i_{k^{t}+1}\right\}\right] \cup\left\{i_{k^{t}}\right\}\right)>\operatorname{ed}\left(\boldsymbol{q}_{x},\left[\mu^{-1}(x) \backslash\left\{i_{k^{t}+1}\right\}\right] \cup\{j\}\right) .
$$

A contradiction. If instead $\operatorname{ed}\left(\boldsymbol{q}_{x}, A\right)=\operatorname{ed}\left(\boldsymbol{q}_{x}, \nu^{-1}(x)\right)$ then $j \succ_{x}^{\text {exo }} i_{k^{t}}$, and

$$
\operatorname{ed}\left(\boldsymbol{q}_{x},\left[\mu^{-1}(x) \backslash\left\{i_{k^{t}+1}\right\}\right] \cup\left\{i_{k^{t}}\right\}\right)=\operatorname{ed}\left(\boldsymbol{q}_{x},\left[\mu^{-1}(x) \backslash\left\{i_{k^{t}+1}\right\}\right] \cup\{j\}\right),
$$

which implies that $i_{k^{t}} \notin E_{i_{k^{t}+1}}^{\mu}$.

Suppose $\ell \neq i_{k^{t}}$. There are three subcases:

Case 2.1: $\tau(j) \neq \tau\left(i_{k^{t}}\right), \tau\left(i_{k^{t}+1}\right)$.

If $\tau(\ell)=\tau\left(i_{k^{t}}\right)$ then $\tau(\ell) \neq \tau\left(i_{k^{s}}\right)$ for all $s \neq t$. This implies that $\mu(\ell)=\nu(\ell)$. Since $\mu$ is stable, we have $\left|\left(\mu^{-1}(x)\right)_{\tau\left(i_{k^{t}}\right)}\right| \geq q_{x}^{\tau\left(i_{k^{t}}\right)}$, and

$$
\ell \succsim_{x}^{e x o} i_{k^{t}}
$$

A similar argument for $\ell=i_{k^{t}}$ leads to contradiction.

If $\tau(\ell)=\tau\left(i_{k^{t}+1}\right)$ then clearly $\tau(\ell) \neq \tau\left(i_{k^{t}}\right)$ and by the claim $\tau\left(i_{k^{t}+1}\right) \neq \tau\left(i_{k^{s}}\right)$ for any $s \neq t$, which implies that $\ell \in\{i \in N \mid \mu(i)=\nu(i)\}$. Since $\operatorname{ed}\left(\boldsymbol{q}_{x}, A\right) \leq \operatorname{ed}\left(\boldsymbol{q}_{x}, \nu^{-1}(x)\right)$,

$$
\begin{gathered}
\left(\left|\left(\mu^{-1}(x)\right)_{\tau(\ell)}\right|-2-q_{x}^{\tau(\ell)}\right)^{2}+\left(\left|\left(\mu^{-1}(x)\right)_{\tau(j)}\right|+1-q_{x}^{\tau(j)}\right)^{2} \leq \\
\left(\left|\left(\mu^{-1}(x)\right)_{\tau(\ell)}\right|-1-q_{x}^{\tau(\ell)}\right)^{2}+\left(\left|\left(\mu^{-1}(x)\right)_{\tau(j)}\right|-q_{x}^{\tau(j)}\right)^{2} .
\end{gathered}
$$

Setting $a=\left|\left(\mu^{-1}(x)\right)_{\tau(j)}\right|-q_{x}^{\tau(j)}$, we have $\left|\left(\mu^{-1}(x)\right)_{\tau(\ell)}\right|-q_{x}^{\tau(\ell)} \geq a+2$. On the other hand $\operatorname{ed}\left(\boldsymbol{q}_{x}, \mu^{-1}(x)\right) \leq \operatorname{ed}\left(\boldsymbol{q}_{x},\left[\mu^{-1}(x) \backslash\{\ell\}\right] \cup\{j\}\right)$ implies $\left|\left(\mu^{-1}(x)\right)_{\tau(\ell)}\right|-q_{x}^{\tau(\ell)} \leq a+1$, a contradiction.

So we assume that $\tau(\ell)$ equals neither $\tau\left(i_{k^{t}}\right)$ nor $\tau\left(i_{k^{t}+1}\right)$. Since ed $\left(\boldsymbol{q}_{x},\left[\nu^{-1}(x) \backslash\{\ell\}\right] \cup\right.$ $\{j\}) \leq \operatorname{ed}\left(\boldsymbol{q}_{x}, \nu^{-1}(x)\right)$ and $\operatorname{ed}\left(\boldsymbol{q}_{x},\left[\mu^{-1}(x) \backslash\{\ell\}\right] \cup\{j\}\right) \geq \operatorname{ed}\left(\boldsymbol{q}_{x}, \mu^{-1}(x)\right)$,

$$
\begin{gathered}
\left(\left|\left(\mu^{-1}(x)\right)_{\tau(\ell)}\right|-1-q_{x}^{\tau(\ell)}\right)^{2}+\left(\left|\left(\mu^{-1}(x)\right)_{\tau(j)}\right|-q_{x}^{\tau(j)}\right)^{2}= \\
\left(\left|\left(\mu^{-1}(x)\right)_{\tau(\ell)}\right|-q_{x}^{\tau(\ell)}\right)^{2}+\left(\left|\left(\mu^{-1}(x)\right)_{\tau(j)}\right|+1-q_{x}^{\tau(j)}\right)^{2} .
\end{gathered}
$$

If $\ell=i_{k^{s}}$ for any $s \neq t$, then $i_{k^{s}} \notin E_{i_{k^{s}+1}}^{\mu}$. Otherwise it contradicts the fact that $\mu$ is stable. 
Case 2.2: $\tau(j)=\tau\left(i_{k^{t}}\right)$

First, consider the case $\tau(\ell) \neq \tau\left(i_{k^{t}+1}\right)$. If $\tau(\ell)=\tau(j)$, then $\operatorname{ed}\left(\boldsymbol{q}_{x}, A\right)=\operatorname{ed}\left(\boldsymbol{q}_{x}, \nu^{-1}(x)\right)$ and $j \succ_{x}^{e x o} \ell$. Now if $\ell=i_{k^{s}}$ for some $s \neq t$, then we have $i_{k^{s}} \notin E_{i_{k^{s}+1}}^{\mu}$, whereas if $\ell \neq i_{k^{s}}$ for all $s$, then $\mu$ cannot be stable. Hence we must have $\tau(\ell) \neq \tau(j)$.

We know that $\left|\left(\mu^{-1}(x)\right)_{\tau(j)}\right| \geq q_{x}^{\tau(j)}$. Since ed $\left(\boldsymbol{q}_{x},\left[\nu^{-1}(x) \backslash\{\ell\}\right] \cup\{j\}\right) \leq \operatorname{ed}\left(\boldsymbol{q}_{x}, \nu^{-1}(x)\right)$,

$$
\begin{gathered}
\left(\left|\left(\mu^{-1}(x)\right)_{\tau(\ell)}\right|-1-q_{x}^{\tau(\ell)}\right)^{2}+\left(\left|\left(\mu^{-1}(x)\right)_{\tau(j)}\right|+2-q_{x}^{\tau(j)}\right)^{2} \leq \\
\left(\left|\left(\mu^{-1}(x)\right)_{\tau(\ell)}\right|-q_{x}^{\tau(\ell)}\right)^{2}+\left(\left|\left(\mu^{-1}(x)\right)_{\tau(j)}\right|+1-q_{x}^{\tau(j)}\right)^{2}
\end{gathered}
$$

which implies that whenever $\left|\left(\mu^{-1}(x)\right)_{\tau(j)}\right|-q_{x}^{\tau(j)}=a>0$, we must have

$$
\left|\left(\mu^{-1}(x)\right)_{\tau(\ell)}\right|-q_{x}^{\tau(\ell)} \geq a+2
$$

If $\ell=i_{k^{s}}$ for some $s \neq t$, then

$\operatorname{ed}\left(\boldsymbol{q}_{x},\left[\mu^{-1}(x) \backslash\left\{i_{k^{s}+1}\right\}\right] \cup\left\{i_{k^{s}}\right\}\right)=C+\left(\left|\left(\mu^{-1}(x)\right)_{\tau\left(i_{k^{s}}\right)}\right|-q_{x}^{\tau\left(i_{k^{s}}\right)}\right)^{2}+\left(\left|\left(\mu^{-1}(x)\right)_{\tau(j)}\right|-q_{x}^{\tau(j)}\right)^{2}$

and

$\operatorname{ed}\left(\boldsymbol{q}_{x},\left[\mu^{-1}(x) \backslash\left\{i_{k^{s}+1}\right\}\right] \cup\{j\}\right)=C+\left(\left|\left(\mu^{-1}(x)\right)_{\tau\left(i_{k} s\right.}\right|-1-q_{x}^{\tau\left(i_{k^{s}}\right)}\right)^{2}+\left(\left|\left(\mu^{-1}(x)\right)_{\tau(j)}\right|+1-q_{x}^{\tau(j)}\right)^{2}$

where $C$ is a constant. Thus we conclude that

$$
\operatorname{ed}\left(\boldsymbol{q}_{x},\left[\mu^{-1}(x) \backslash\left\{i_{k^{s}+1}\right\}\right] \cup\left\{i_{k^{s}}\right\}\right)>\operatorname{ed}\left(\boldsymbol{q}_{x},\left[\mu^{-1}(x) \backslash\left\{i_{k^{s}+1}\right\}\right] \cup\{j\}\right),
$$

which means that $i_{k^{s}} \notin E_{i_{k}+1}^{\mu}$.

If $\ell \neq i_{k^{s}}$ for any $s$, then

$$
\operatorname{ed}\left(\boldsymbol{q}_{x}, \mu^{-1}(x)\right)=C+\left(\left|\left(\mu^{-1}(x)\right)_{\tau(\ell)}\right|-q_{x}^{\tau(\ell)}\right)^{2}+\left(\left|\left(\mu^{-1}(x)\right)_{\tau(j)}\right|-q_{x}^{\tau(j)}\right)^{2},
$$

and

$\operatorname{ed}\left(\boldsymbol{q}_{x},\left[\mu^{-1}(x) \backslash\{\ell\}\right] \cup\{j\}\right)=C+\left(\left|\left(\mu^{-1}(x)\right)_{\tau(\ell)}\right|-1-q_{x}^{\tau(\ell)}\right)^{2}+\left(\left|\left(\mu^{-1}(x)\right)_{\tau(j)}\right|+1-q_{x}^{\tau(j)}\right)^{2}$

where $C$ is a constant. Thus we conclude that

$$
\operatorname{ed}\left(\boldsymbol{q}_{x}, \mu^{-1}(x)\right)>\operatorname{ed}\left(\boldsymbol{q}_{x},\left[\mu^{-1}(x) \backslash\{\ell\}\right] \cup\{j\}\right),
$$

which contradicts the fact that $\mu$ is stable. 
Next consider $\tau(\ell)=\tau\left(i_{k^{t}+1}\right)$. Since ed $\left(\boldsymbol{q}_{x},\left[\mu^{-1}(x) \backslash\{\ell\}\right] \cup\{j\}\right)=\operatorname{ed}\left(\boldsymbol{q}_{x}, \nu^{-1}(x)\right)$ and $\operatorname{ed}\left(\boldsymbol{q}_{x},\left[\nu^{-1}(x) \backslash\{\ell\}\right] \cup\{j\}\right) \leq \operatorname{ed}\left(\boldsymbol{q}_{x}, \nu^{-1}(x)\right)$,

$$
\begin{aligned}
& \left(\left|\left(\mu^{-1}(x)\right)_{\tau\left(i_{k^{t}+1}\right)}\right|-2-q_{x}^{\tau\left(i_{k^{t}+1}\right)}\right)^{2}+\left(\left|\left(\mu^{-1}(x)\right)_{\tau(j)}\right|+2-q_{x}^{\tau(j)}\right)^{2} \leq \\
& \left(\left|\left(\mu^{-1}(x)\right)_{\tau\left(i_{k^{t}+1}\right)}\right|-1-q_{x}^{\tau\left(i_{k^{t}+1}\right)}\right)^{2}+\left(\left|\left(\mu^{-1}(x)\right)_{\tau(j)}\right|+1-q_{x}^{\tau(j)}\right)^{2},
\end{aligned}
$$

which implies that whenever $\left|\left(\mu^{-1}(x)\right)_{\tau(j)}\right|-q_{x}^{\tau(j)}=a>0$, we must have

$$
\left|\left(\mu^{-1}(x)\right)_{\tau(\ell)}\right|-q_{x}^{\tau(\ell)} \geq a+\frac{3}{2} .
$$

Then

$$
\operatorname{ed}\left(\boldsymbol{q}_{x}, \mu^{-1}(x)\right)=C+\left(\left|\left(\mu^{-1}(x)\right)_{\tau(\ell)}\right|-q_{x}^{\tau(\ell)}\right)^{2}+\left(\left|\left(\mu^{-1}(x)\right)_{\tau(j)}\right|-q_{x}^{\tau(j)}\right)^{2}
$$

and

$\operatorname{ed}\left(\boldsymbol{q}_{x},\left[\mu^{-1}(x) \backslash\{\ell\}\right] \cup\{j\}\right)=C+\left(\left|\left(\mu^{-1}(x)\right)_{\tau(\ell)}\right|-1-q_{x}^{\tau(\ell)}\right)^{2}+\left(\left|\left(\mu^{-1}(x)\right)_{\tau(j)}\right|+1-q_{x}^{\tau(j)}\right)^{2}$

where $C$ is a constant. Thus we conclude that

$$
\operatorname{ed}\left(\boldsymbol{q}_{x}, \mu^{-1}(x)\right)>\operatorname{ed}\left(\boldsymbol{q}_{x},\left[\mu^{-1}(x) \backslash\{\ell\}\right] \cup\{j\}\right)
$$

which contradicts the fact that $\mu$ is stable.

Case 2.3: $\tau(j)=\tau\left(i_{k^{t}+1}\right)$

Then $\operatorname{ed}\left(\boldsymbol{q}_{x}, \mu^{-1}(x)\right)=\operatorname{ed}\left(\boldsymbol{q}_{x},\left[\mu^{-1}(x) \backslash\left\{i_{k^{t}+1}\right\}\right] \cup\{j\}\right)$. Since $i_{k^{t}} \in E_{i_{k^{t}+1}}^{\mu}$ and $\mu$ is stable, we must have ed $\left(\boldsymbol{q}_{x},\left[\mu^{-1}(x) \backslash\left\{i_{k^{t}+1}\right\}\right] \cup\left\{i_{k^{t}}\right\}\right)=\operatorname{ed}\left(\boldsymbol{q}_{x},\left[\mu^{-1}(x) \backslash\left\{i_{k^{t}+1}\right\}\right] \cup\{j\}\right)$ and $i_{k^{t}} \succsim^{e x o} j$. This implies that

$$
\operatorname{ed}\left(\boldsymbol{q}_{x}, \mu^{-1}(x)\right)=\operatorname{ed}\left(\boldsymbol{q}_{x}, \nu^{-1}(x)\right)
$$

Furthermore,

$$
\operatorname{ed}\left(\boldsymbol{q}_{x},\left[\nu^{-1}(x) \backslash\{\ell\}\right] \cup\{j\}\right)=\operatorname{ed}\left(\boldsymbol{q}_{x}, \mu^{-1}(x)\right),
$$

for otherwise

$$
\mu^{-1}(x) \notin \mathcal{A}_{x}\left(\mu^{-1}(x) \cup\left\{i_{k^{1}}, \cdots, i_{k^{m}}\right\} \cup\{j\}\right) .
$$

This implies that $\ell=i_{k^{s}}$ for some $s \neq k$. Thus, we have

$$
\left.\left.\operatorname{ed}\left(\boldsymbol{q}_{x}, \mu^{-1}(x) \backslash\left\{i_{k^{s}+1}\right\}\right] \cup\{j\}\right)=\operatorname{ed}\left(\boldsymbol{q}_{x}, \nu^{-1}(x) \backslash\left\{i_{k^{s}+1}\right\}\right] \cup\left\{i_{k^{s}}\right\}\right)
$$


$j \succ^{\text {exo }} i_{k^{s}}$ implies that $i_{k^{s}} \notin E_{i_{k^{s}+1}}^{\mu}$.

Case 3: For all $t, \tau\left(i_{k^{t}}\right)=\tau\left(i_{k^{t}+1}\right)$.

Suppose $\ell=i_{k^{t}}$ for some $t$. When $\operatorname{ed}\left(\boldsymbol{q}_{x}, A\right)=\operatorname{ed}\left(\boldsymbol{q}_{x}, \nu^{-1}(x)\right), j \succ_{x}^{\text {exo }} i_{k^{t}}$. Since

$$
\operatorname{ed}\left(\boldsymbol{q}_{x},\left[\mu^{-1}(x) \backslash\left\{i_{k^{t}+1}\right\}\right] \cup\left\{i_{k^{t}}\right\}\right)=\operatorname{ed}\left(\boldsymbol{q}_{x}, \mu^{-1}(x)\right)=\operatorname{ed}\left(\boldsymbol{q}_{x}, \nu^{-1}(x)\right)=\operatorname{ed}\left(\boldsymbol{q}_{x}, A\right),
$$

it must be that

$$
\operatorname{ed}\left(\boldsymbol{q}_{x},\left[\mu^{-1}(x) \backslash\left\{i_{k^{t}+1}\right\}\right] \cup\left\{i_{k^{t}}\right\}\right)=\operatorname{ed}\left(\boldsymbol{q}_{x},\left[\mu^{-1}(x) \backslash\left\{i_{k^{t}+1}\right\}\right] \cup\{j\}\right) .
$$

$i_{k^{t}} \in E_{i_{k^{t}+1}}^{\mu}$ and $j \in \mathcal{E}_{i_{k^{t}+1}}^{\mu}$ imply that $i_{k^{t}} \succsim_{x}^{\text {exo }} j$. A contradiction.

When $\operatorname{ed}\left(\boldsymbol{q}_{x}, A\right)<\operatorname{ed}\left(\boldsymbol{q}_{x}, \nu^{-1}(x)\right)$,

$$
\operatorname{ed}\left(\boldsymbol{q}_{x},\left[\mu^{-1}(x) \backslash\left\{i_{k^{t}+1}\right\}\right] \cup\{j\}\right)<\operatorname{ed}\left(\boldsymbol{q}_{x},\left[\mu^{-1}(x) \backslash\left\{i_{k^{t}+1}\right\}\right] \cup\left\{i_{k^{t}}\right\}\right)
$$

which implies $i_{k^{t}} \notin E_{i_{k^{t}+1}}^{\mu}$, a contradiction.

Suppose instead that $\ell \neq i_{k^{t}}$ for all $t$. Then $\mu(\ell)=\nu(\ell)=x$. If $\operatorname{ed}\left(\boldsymbol{q}_{x}, A\right)=$ $\operatorname{ed}\left(\boldsymbol{q}_{x}, \nu^{-1}(x)\right)$, then $\operatorname{ed}\left(\boldsymbol{q}_{x}, A\right)=\operatorname{ed}\left(\boldsymbol{q}_{x}, \mu^{-1}(x)\right)=\operatorname{ed}\left(\boldsymbol{q}_{x},\left[\mu^{-1}(x) \backslash\{\ell\}\right] \cup\{j\}\right)$. Since $j \succ_{x}^{e x o} \ell$ implies that

$$
\left[\mu^{-1}(x) \backslash\{\ell\}\right] \cup\{j\} \succ_{x} \mu^{-1}(x)
$$

contradicting the fact that $\mu$ is stable. If, on the other hand, $\operatorname{ed}\left(\boldsymbol{q}_{x}, A\right)<\operatorname{ed}\left(\boldsymbol{q}_{x}, \nu^{-1}(x)\right)$, then $\operatorname{ed}\left(\boldsymbol{q}_{x},\left[\mu^{-1}(x) \backslash\{\ell\}\right] \cup\{j\}\right)<\operatorname{ed}\left(\boldsymbol{q}_{x}, \mu^{-1}(x)\right)$, which implies that $\mu$ is not stable; again a contradiction.

\section{Proof of Proposition 6}

$(\Leftarrow)$ Already known by Proposition 3 .

$(\Rightarrow)$ Suppose $\mu$ is stable and admits a PSIC denoted by

$$
i_{0} \rightarrow i_{1} \rightarrow \cdots \rightarrow i_{n-1} \rightarrow i_{0}
$$

Let $\nu$ be the assignment obtained by carrying out this PSIC. If $\mu\left(i_{0}\right), \ldots, \mu\left(i_{n-1}\right)$ are distinct, then this PSIC boils down to a SIC (in the sense of Erdil and Ergin, 2008), and therefore $\nu$ is stable. Now, suppose there is some school $x$ which shows up more than once in the above PSIC. Say $x$ appears $m$ times in the PSIC, and enumerate the 
students who move from their matched school under $\mu$ to $x$ as part of this PSIC as $i_{k^{t}}$ for $t \in\{1, \ldots, m\}$. Thus $\nu\left(i_{k^{t}}\right)=\mu\left(i_{k^{t}+1}\right)=x$.

We will verify that $\nu$ is stable by showing that for each such $x$ in the PSIC,

$$
\nu^{-1}(x) \text { is a chosen subset from } S \text { whenever } \nu^{-1}(x) \subseteq S \subseteq \mathcal{E}_{x}^{\nu} \cup \nu^{-1}(x) .
$$

First, stability of $\mu$ implies $\mu^{-1}(x)$ is a chosen subset from $\mu^{-1}(x) \cup \mathcal{E}_{x}^{\mu}$, and therefore there exists $\pi^{0} \in \Pi$ such that

$$
\mathcal{A}^{\pi^{0}}\left(\mu^{-1}(x) \cup \mathcal{E}_{x}^{\mu}\right)=\mu^{-1}(x)
$$

Let us enumerate the $q-m$ elements in $\mu^{-1}(x) \cap \nu^{-1}(x)$ in their order of being selected from $\mu^{-1}(x) \cup \mathcal{E}_{x}^{\mu}$ so $j_{1}$ is selected earlier than $j_{2}$ who was selected earlier than $j_{3}$ and so on. Let $\succ^{t}$ be the criterion used in $\mathcal{A}^{\pi^{0}}$ when selecting $j_{t}$. Then, it must be the case that

$$
j_{1} \text { is the highest } \succ^{1} \text { ranked student in }\left[\left(\mu^{-1}(x) \cap \nu^{-1}(x)\right) \cup \mathcal{E}_{x}^{\mu}\right],
$$

and for $t=2, \ldots, q-m$,

$j_{t}$ is the highest $\succ^{t}$ ranked student in $\left[\left(\mu^{-1}(x) \cap \nu^{-1}(x)\right) \cup \mathcal{E}_{x}^{\mu}\right] \backslash\left\{j_{1}, \ldots, j_{t-1}\right\}$

Note that $\left(\mu^{-1}(x) \cap \nu^{-1}(x)\right) \cup \mathcal{E}_{x}^{\mu}=\nu^{-1}(x) \cup \mathcal{E}_{x}^{\nu}$, so we must have

$$
j_{t} \text { is the highest } \succ^{t} \text { ranked student in }\left[\nu^{-1}(x) \cup \mathcal{E}_{x}^{\nu}\right] \backslash\left\{j_{1}, \ldots, j_{t-1}\right\} \text {. }
$$

Next, by the definition of PSIC, we know for each $t \in\{1, \ldots, m\}$ that

$$
\left\{i_{k^{t}}\right\} \cup\left[\mu^{-1}(x) \backslash\left\{i_{k^{t}+1}\right\}\right] \text { is chosen from }\left(\mathcal{E}_{x}^{\mu} \cup \mu^{-1}(x)\right) \backslash\left\{i_{k^{t}+1}\right\} .
$$

That is, there exists $\pi^{t} \in \Pi$ such that

$$
\mathcal{A}^{\pi^{t}}\left(\left(\mathcal{E}_{x}^{\mu} \cup \mu^{-1}(x)\right) \backslash\left\{i_{k^{t}+1}\right\}\right)=\left\{i_{k^{t}}\right\} \cup\left[\mu^{-1}(x) \backslash\left\{i_{k^{t}+1}\right\}\right]
$$

Then, there must be a criterion $\succ$ which features in $\pi^{t}$ such that

$$
i_{k^{t}} \succ j \quad \text { for all } j \in \mathcal{E}_{x}^{\mu} \backslash\left\{i_{k^{t}}\right\} .
$$

Denote that criterion by $\succ^{q-m+t}$.

Finally define $\pi^{*} \in \Pi$ by setting $\pi^{*}(\ell)=\succ^{\ell}$ for each $\ell \in\{1, \ldots, q\}$. Now given any set $S$ of applicants with $\nu^{-1}(x) \subseteq S \subseteq \mathcal{E}_{x}^{\nu} \cup \nu^{-1}(x)$, we will have

$$
A^{\pi^{*}}(S)=\nu^{-1}(x) \text {. }
$$




\section{References}

[1] Abdulkadiroğlu, Atila. 2005. "College admissions with affirmative action." International Journal of Game Theory 33 (4): 535-549.

[2] Abdulkadiroğlu, Atila, Parag A. Pathak, and Alvin E. Roth. 2009. "Strategy-proofness versus efficiency in matching with indifferences: redesigning the NYC high school match." American Economic Review 99 (5): 1954-1978.

[3] Abdulkadiroğlu, Atila, Parag A. Pathak, Alvin E. Roth, and Tayfun Sönmez. 2005. "The Boston public school match." American Economic Review 95 (2): $368-371$.

[4] Abdulkadiroğlu, Atila, and Tayfun Sönmez. 2003. "School choice: a mechanism design approach." American Economic Review 93 (3): 729-747.

[5] Cormen, Thomas H., Charles E. Leiserson, Ronald L. Rivest, and Clifford Stein. 2001. Introduction to Algorithms. Cambridge: MIT Press.

[6] Dur, Umut, Scott D. Kominers, Parag A. Pathak, and Tayfun Sönmez. 2018. "Reserve Design: Unintended Consequences and The Demise of BostonÕs Walk Zones." Journal of Political Economy 126 (6): 2457-2479.

[7] Echenique, Federico. 2007. "Counting combinatorial choice rules." Games and Economic Behavior. Vol. 58 (February 2007), 231-245

[8] Echenique, Federico, and M. Bumin Yenmez. 2015. "How to control controlled school choice." American Economic Review 105 (8): 2679-2694.

[9] Ehlers, Lars. 2007. "Respecting Priorities when Assigning Students to Schools." mimeo.

[10] Ehlers, Lars, and Aytek Erdil. 2010. "Efficient assignment respecting priorities." Journal of Economic Theory 145 (3): 1269-1282.

[11] Ehlers, Lars, Isa E. Hafalir, M. Bumin Yenmez, and Muhammed A. Yildirim. 2014. "School Choice with Controlled Choice Constraints: Hard Bounds versus Soft Bounds." Journal of Economic Theory 153: 648-683. 
[12] Erdil, Aytek. 2014. "Strategy-proof stochastic assignment." Journal of Economic Theory 151: 146-162.

[13] Erdil, Aytek, and Haluk Ergin. 2008. "What's the matter with tie-breaking? Improving efficiency in school choice." American Economic Review 98 (3): 669-689.

[14] Ergin, Haluk I. 2002. "Efficient resource allocation on the basis of priorities." Econometrica 70 (6): 2489-2497.

[15] Gale, David, and Lloyd S. Shapley. 1962. "College admissions and the stability of marriage." American Mathematical Monthly 69 (1): 9-15.

[16] Hafalir, Isa E., M. Bumin Yenmez, and Muhammed A. Yildirim. 2013. "Effective Affirmative Action in School Choice." Theoretical Economics 8 (2): 352363.

[17] Han, Xiang. 2018. "Stable and efficient resource allocation under weak priorities." Games and Economic Behavior 107 (1): 9-20.

[18] Hatfield, John William, and Paul R. Milgrom. 2005. "Matching with Contracts." American Economic Review 95 (4): 913-935.

[19] Kelso, Alexander S. Jr., and Vincent P. Crawford. 1982. "Job Matching, Coalition Formation, and Gross Substitutes." Econometrica 50 (6): 1483-1504.

[20] Kojima, Fuhito. 2012. "School Choice: Impossibilities for Affirmative Action." Games and Economic Behavior 75 (2): 685-693.

[21] Kominers, Scott D., and Tayfun Sönmez. 2016. "Matching with Slot-Specific Priorities: Theory." Theoretical Economics 11: 683-710.

[22] Roth, Alvin E. 1984a. "Stability and polarization of interests in job matching." Econometrica 52 (1): 47-58.

[23] Roth, Alvin E. 1984b. "The Evolution of the Labor Market for Medical Interns and Residents: A Case Study in Game Theory." Journal of Political Economy 92 (6): 991-1016. 
[24] Roth, Alvin E. 1985. "The College Admissions Problem is Not Equivalent to the Marriage Problem." Journal of Economic Theory 36 (2): 277-288.

[25] Roth, Alvin E. 2008. "Deferred acceptance algorithms: history, theory, practice, and open questions." International Journal of Game Theory 36 (3): 537-569.

[26] Roth, Alvin E., and Marilda A. O. Sotomayor. 1990. Two-Sided Matching: A Study in Game-Theoretic Modelling and Analysis. Cambridge, UK: Cambridge University Press.

[27] Sotomayor, Marilda. 1999. "Three remarks on the many-to-many stable matching problem." Mathematical Social Sciences, 38 (1): 55-70. 


\section{Online Appendix: Remarks}

Remark 1 When $\mathcal{A}_{x}$ is strict, AM is equivalent to RM. When there are ties, i.e., when $\mathcal{A}_{x}$ is multivalued, neither condition necessarily implies the other.

Proof. First, suppose $\mathcal{A}_{x}$ is strict.

We say $\mathcal{A}_{x}$ is admission monotonic (AM) if given any $S, T \subseteq N$ with $S \subseteq T$ and $T^{\prime} \in \mathcal{A}_{x}(T)$, we have $T^{\prime} \cap S \subseteq S^{\prime}$ for some $S^{\prime} \in \mathcal{A}_{x}(S)$.

In a similar spirit, we say $\mathcal{A}_{x}$ is rejection monotonic (RM) if given any $S, T \subseteq N$ with $S \subseteq T$ and $S^{\prime \prime} \in \mathcal{R}_{x}(S)$, we have $S^{\prime \prime} \subseteq T^{\prime \prime}$ for some $T^{\prime \prime} \in \mathcal{R}_{x}(T)$.

$\underline{\mathrm{AM} \Rightarrow \mathrm{RM}}$. Suppose $S \subseteq T$ and $S^{\prime \prime} \in \mathcal{R}_{x}(S)$. Then, denoting $S^{\prime}=S \backslash S^{\prime \prime}$, we must have $S^{\prime} \in \mathcal{A}_{x}(S)$. Since $\mathcal{A}_{x}$ is strict, we have $S^{\prime}=\left\{\mathcal{A}_{x}(S)\right\}$. Let $\mathcal{A}_{x}(T)=\left\{T^{\prime}\right\}$, so denoting $T^{\prime \prime}=T \backslash T^{\prime}$, we have $\mathcal{R}_{x}(T)=\left\{T^{\prime \prime}\right\}$. AM implies that $T^{\prime} \cap S \subseteq S^{\prime}$. Taking the complement of this last expression in $S$ yields $S \backslash T^{\prime} \supseteq S^{\prime \prime}$. Combining this with the fact that $S \backslash T^{\prime} \subseteq T \backslash T^{\prime}=T^{\prime \prime}$ yields $S^{\prime \prime} \subseteq T^{\prime \prime}$.

$\underline{\mathrm{AM}} \Leftarrow \mathrm{RM}$. Suppose $S \subseteq T$ and $T^{\prime} \in \mathcal{A}_{x}(T)$. Let $\left\{S^{\prime}\right\}=\mathcal{A}_{x}(S)$, so $S^{\prime \prime}=S \backslash S^{\prime}=$ $\mathcal{R}_{x}(S)$. RM implies $S^{\prime \prime} \subseteq T^{\prime \prime}=T \backslash T^{\prime}$. Removing both sides of this last inclusion from $S$ yields $S^{\prime} \supseteq S \backslash\left(T \backslash T^{\prime}\right)=S \cap T^{\prime}$.

Next, we show that for multivalued admission rules, neither of AM and RM implies the other.

$\underline{\mathrm{AM}} \nRightarrow \mathrm{RM}$. A multivalued admission rule $\mathcal{A}_{x}$ which is admission monotonic, but not rejection monotonic:

Suppose there are four students $i_{1}, i_{2}, i_{3}, i_{4}$, and a school $x$ with two seats and the following priority ranking: $\left\{i_{1}, i_{4}\right\} \succ_{x}\left\{i_{1}, i_{2}\right\} \sim_{x}\left\{i_{1}, i_{3}\right\} \sim_{x}\left\{i_{2}, i_{3}\right\} \sim_{x}\left\{i_{2}, i_{4}\right\} \sim_{x}$ $\left\{i_{3}, i_{4}\right\}$. It is readily verified that $\succsim_{x}$ leads to an admission rule $\mathcal{A}_{x}$ which is AM. To see that $\mathcal{A}_{x}$ is not $\mathrm{RM}$, note that

$$
\mathcal{R}_{x}\left(\left\{i_{1}, i_{2}, i_{3}\right\}\right)=\left\{\left\{i_{1}\right\},\left\{i_{2}\right\},\left\{i_{3}\right\}\right\}
$$

but

$$
\mathcal{R}_{x}\left(\left\{i_{1}, i_{2}, i_{3}, i_{4}\right\}\right)=\left\{\left\{i_{2}, i_{3}\right\}\right\}
$$

hence

$$
\left\{i_{1}\right\} \nsubseteq\left\{i_{2}, i_{3}\right\}
$$


$\underline{\mathrm{AM}} \nLeftarrow \mathrm{RM}$. A multivalued admission rule $\mathcal{A}_{x}$ which is rejection monotonic, but not admission monotonic: Suppose there are five students $\left\{i_{1}, i_{2}, i_{3}, i_{4}, i_{5}\right\}$, and a school $x$ with two seats and the following priority ranking: $\left\{i_{1}, i_{2}\right\} \sim_{x}\left\{i_{3}, i_{4}\right\} \succ_{x}\left\{i_{1}, i_{3}\right\} \sim_{x}$ $\left\{i_{1}, i_{4}\right\} \sim_{x}\left\{i_{1}, i_{5}\right\} \sim_{x}\left\{i_{2}, i_{3}\right\} \sim_{x}\left\{i_{2}, i_{4}\right\} \succ_{x}\left\{i_{2}, i_{5}\right\} \sim_{x}\left\{i_{3}, i_{5}\right\} \sim_{x}\left\{i_{4}, i_{5}\right\}$.

It is straightforward to verify that $\succsim_{x}$ leads to an admission rule $\mathcal{A}_{x}$ which is RM. On the other hand AM fails, because

$$
\mathcal{A}_{x}\left(\left\{i_{1}, i_{2}, i_{3}, i_{4}\right\}\right)=\left\{\left\{i_{1}, i_{2}\right\},\left\{i_{3}, i_{4}\right\}\right\}
$$

but

$$
\mathcal{A}_{x}\left(\left\{i_{1}, i_{2}, i_{3}\right\}\right)=\left\{\left\{i_{1}, i_{2}\right\}\right\}
$$

hence

$$
\left\{i_{3}\right\}=\left\{i_{3}, i_{4}\right\} \cap\left\{i_{1}, i_{2}, i_{3}\right\} \nsubseteq\left\{i_{1}, i_{2}\right\}
$$

Remark 2 An acceptant admission rule $\mathcal{A}$ is rejection monotonic if and only if for every $S \subseteq T$, and for each $S^{\prime} \in \mathcal{A}_{x}(S)$, there exists $T^{\prime} \in \mathcal{A}_{x}(T)$ such that $T^{\prime} \cap S \subseteq S^{\prime}$.

Proof. In order to verify this equivalence, first recall that the rejection rule associated with $\mathcal{A}$ is defined as $\mathcal{R}(S)=\left\{S^{\prime \prime} \subseteq S \mid S^{\prime \prime}=S \backslash S^{\prime}\right.$ for some $\left.S^{\prime} \in \mathcal{A}(S)\right\}$.

$(\Rightarrow)$ : let $S^{\prime \prime} \in \mathcal{R}_{x}(S)$. Then $S^{\prime \prime}=S \backslash S^{\prime}$ for some $S^{\prime} \in \mathcal{A}(S)$, and hence $T^{\prime} \cap S \subseteq S^{\prime}$ for some $T^{\prime} \in \mathcal{A}(T)$. Taking complements in $T$, we get

$$
T \backslash\left(T^{\prime} \cap S\right) \supseteq T \backslash S^{\prime} \supseteq S \backslash S^{\prime}=S^{\prime \prime}
$$

Set $T^{\prime \prime}=T \backslash T^{\prime}$. Since $T \backslash\left(T^{\prime} \cap S\right)=\left(T \backslash T^{\prime}\right) \cup(T \backslash S)$, the last inclusion yields

$$
\begin{array}{rll}
T^{\prime \prime} \cup(T \backslash S) & \supseteq S^{\prime \prime} \\
\left(T^{\prime \prime} \cup(T \backslash S)\right) \cap S & \supseteq S^{\prime \prime} \cap S=S^{\prime \prime} \\
T^{\prime \prime} \cap S & \supseteq S^{\prime \prime}
\end{array}
$$

which implies $T^{\prime \prime} \supseteq S^{\prime \prime}$.

$(\Leftarrow)$ : If $S^{\prime} \in \mathcal{A}(S)$, then $S^{\prime}=S \backslash S^{\prime \prime}$ for some $S^{\prime \prime} \in \mathcal{R}(S)$. Since we assume $S^{\prime \prime} \subseteq T^{\prime \prime}$ for some $T^{\prime \prime} \in \mathcal{R}(T)$, by taking complements in $T$ we obtain

$$
T \backslash S^{\prime \prime} \supseteq T \backslash T^{\prime \prime}=T^{\prime}
$$


Intersection with $S$ yields

$$
\begin{aligned}
\left(T \backslash S^{\prime \prime}\right) \cap S & \supseteq T^{\prime} \cap S \\
T \cap\left(S^{\prime \prime}\right)^{c} \cap S & \supseteq T^{\prime} \cap S \\
S \cap\left(S^{\prime \prime}\right)^{c} & \supseteq T^{\prime} \cap S \\
S^{\prime} & \supseteq T^{\prime} \cap S .
\end{aligned}
$$

Remark 3 Given a substitutable priority structure, arbitrarily breaking ties and then applying the DA algorithm does not necessarily lead to a stable assignment.

Proof. Consider four students 1,2,3,4, and two schools $x$ and $y$. Suppose that school $x$ has two seats, whereas $y$ has one. Suppose also that school $y$ finds only student 2 acceptable, while school $x$ has the following acceptant admission rule $\mathcal{A}_{x}$ :

$$
\begin{aligned}
& \mathcal{A}_{x}(\{1,2,3,4\})=\{\{1,2\},\{3,4\}\} \\
& \mathcal{A}_{x}(\{1,2,3\})=\{\{1,2\},\{2,3\}\} \\
& \mathcal{A}_{x}(\{1,2,4\})=\{\{1,2\},\{1,4\}\} \\
& \mathcal{A}_{x}(\{1,3,4\})=\{\{1,3\},\{3,4\}\} \\
& \mathcal{A}_{x}(\{2,3,4\})=\{\{2,3\},\{2,4\},\{3,4\}\}
\end{aligned}
$$

It is straightforward to verify that $\mathcal{A}_{x}$ is substitutable. One possible tie-breaking is

$$
\begin{aligned}
& \mathcal{A}_{x}^{\prime}(\{1,2,3,4\})=\{3,4\} \\
& \mathcal{A}_{x}^{\prime}(\{1,2,3\})=\{2,3\} \\
& \mathcal{A}_{x}^{\prime}(\{1,2,4\})=\{1,2\} \\
& \mathcal{A}_{x}^{\prime}(\{1,3,4\})=\{1,3\} \\
& \mathcal{A}_{x}^{\prime}(\{2,3,4\})=\{2,4\}
\end{aligned}
$$

Suppose all students find both schools acceptable, students 1,2 , and 3 prefer $x$ to $y$, and student 4 prefers $y$ to $x$. Then the deferred acceptance algorithm applied to these preferences and the priority structure $\mathcal{A}^{\prime}$ results in:

$$
\mu=\left(\begin{array}{llll}
1 & 2 & 3 & 4 \\
1 & x & 3 & x
\end{array}\right)
$$


Let us say an assignment $\mu$ is blocked by $i$ and $x$ if $x P_{i} \mu(i), i \notin \mu^{-1}(x)$, and $i \in A$ for all $A \in \mathcal{A}_{x}\left(\mu^{-1}(x) \cup\{i\}\right)$.

First note that $\mu$ is not stable with respect to $\mathcal{A}$, because it is blocked by 1 and $x$. As for $\mathcal{A}^{\prime}$, there does not even exist a stable assignment. In order to see this, note that stability implies that both seats at $x$ must be occupied because all students are acceptable to $x$, and three students top rank $x$.

If 2 is not assigned to $x$, then there are three possibilities as to who ends up at $x$ : (i) 1 and 3; (ii) 1 and 4; or (iii) 3 and 4. However, according to the admission rule $\mathcal{A}_{x}^{\prime}$, all such assignments would be blocked by 2 and $x$. Thus 2 must be assigned to $x$. Now there are three possibilities as to who ends up at $x$ : (i) 2 and 1; (ii) 2 and 3; or (iii) 2 and 4. The first one is blocked by 3 , the second one is blocked by 4 , and the third one is blocked by 1 .

Remark 4 A substitutable admission rule does not necessarily have a substitutable tiebreaking.

Proof. Suppose a school $x$ with two seats is facing five students $1,2,3,4,5$. Omitting irrelevant sets, its admission rule $\mathcal{A}_{x}$ is described as follows

\begin{tabular}{c|c} 
Applicants & Admissible subsets \\
\hline$\{1,2,3,4,5\}$ & $\{1,2\},\{3,5\},\{4,5\}$ \\
$\{1,3,4,5\}$ & $\{1,4\},\{3,5\},\{4,5\}$ \\
$\{2,3,4,5\}$ & $\{2,3\},\{3,5\},\{4,5\}$ \\
$\{3,4,5\}$ & $\{3,5\},\{4,5\}$ \\
$\{1,2,4,5\}$ & $\{1,2\},\{4,5\}$ \\
$\{1,2,3,4\}$ & $\{1,2\},\{1,3\},\{2,4\}$ \\
$\{1,2,4\}$ & $\{1,2\},\{2,4\}$ \\
$\{1,2,3,5\}$ & $\{1,2\},\{3,5\}$ \\
$\{1,2,3\}$ & $\{1,2\},\{1,3\}$
\end{tabular}

It is straightforward to verify that $\mathcal{A}$ is acceptant and substitutable. Now, let $f$ be a tie-breaking of $\mathcal{A}_{x}$, i.e., $f(S) \in \mathcal{A}_{x}(S)$ for all $S \subseteq N=\{1,2,3,4,5\}$. There are three possibilities for $f(N)$, namely $\{1,2\},\{3,5\}$, or $\{4,5\}$.

(1) If $f(N)=\{1,2\}$, then substitutability of $f$ would imply

$$
f(\{1,3,4,5\})=\{1,4\} \quad \text { and } \quad f(\{2,3,4,5\})=\{2,3\}
$$


Again, appealing to substitutability of $f$, we must have

$$
f(\{3,4,5\})=\{3,4\}
$$

which contradicts with the fact that $\mathcal{A}(\{3,4,5\})=\{\{3,5\},\{4,5\}\}$.

(2) If $f(N)=\{3,5\}$, then substitutability of $f$ would imply

$$
f(\{1,2,4,5\})=\{4,5\} \quad \text { and } \quad f(\{1,2,3,4\})=\{1,3\}
$$

Again, appealing to substitutability of $f$, we must have

$$
f(\{1,2,4\})=\{1,4\}
$$

which contradicts with the fact that $\mathcal{A}(\{1,2,4\})=\{\{1,2\},\{2,4\}\}$.

(3) If $f(N)=\{4,5\}$, then substitutability of $f$ would imply

$$
f(\{1,2,3,4\})=\{2,4\} \quad \text { and } \quad f(\{1,2,3,5\})=\{3,5\}
$$

Again, appealing to substitutability of $f$, we must have

$$
f(\{1,2,3\})=\{2,3\}
$$

which contradicts with the fact that $\mathcal{A}(\{1,2,3\})=\{\{1,2\},\{1,3\}\}$.

Remark 5 A PSIC might not preserve stability, even when each student in the cycle can replace the next one while preserving stability ${ }^{20}$

Proof. Let $N=\left\{i_{1}, i_{2}, i_{3}, i_{4}, i_{5}\right\}$. Suppose we have two schools $x$ and $y$ with $q_{x}=2$, $q_{y}=2$. Students' preferences are:

\begin{tabular}{c|c|c|c|c}
$R_{i_{1}}$ & $R_{i_{2}}$ & $R_{i_{3}}$ & $R_{i_{4}}$ & $R_{i_{5}}$ \\
\hline$x$ & $y$ & $x$ & $y$ & $x$ \\
$y$ & $x$ & $y$ & $x$ &
\end{tabular}

Consider the admission rules $\mathcal{A}_{x}$ and $\mathcal{A}_{y}$ implied, respectively, by $\succsim_{x}$ and $\succsim_{y}$ below:

\begin{tabular}{c|c}
$\succsim_{x}$ & $\succsim_{y}$ \\
\hline$\left\{i_{2}, i_{4}\right\}$ & $\left\{i_{1}, i_{2}\right\},\left\{i_{1}, i_{3}\right\},\left\{i_{2}, i_{4}\right\},\left\{i_{3}, i_{4}\right\}$ \\
$\left\{i_{1}, i_{4}\right\},\left\{i_{2}, i_{3}\right\},\left\{i_{2}, i_{5}\right\},\left\{i_{4}, i_{5}\right\}$ & $\left\{i_{1}, i_{4}\right\},\left\{i_{2}, i_{3}\right\}$ \\
$\left\{i_{1}, i_{5}\right\},\left\{i_{3}, i_{5}\right\}$ & the rest \\
$\left\{i_{1}, i_{2}\right\},\left\{i_{1}, i_{3}\right\},\left\{i_{3}, i_{4}\right\}$ &
\end{tabular}

\footnotetext{
${ }^{20}$ This is in contrast with the case of responsive priorities studied in Erdil and Ergin (2008).
} 
The priority structure is acceptant and substitutable. It is straightforward to verify that

$$
\mu=\left(\begin{array}{ccccc}
i_{1} & i_{2} & i_{3} & i_{4} & i_{5} \\
y & x & y & x & i_{5}
\end{array}\right)
$$

is stable.

Now, consider the following cycle

$$
i_{1} \rightarrow i_{2} \rightarrow i_{3} \rightarrow i_{4} \rightarrow i_{1}
$$

in which each student can replace the next, because

$$
\begin{aligned}
& \left\{i_{1}, i_{4}\right\} \in \mathcal{A}_{x}\left(\left\{i_{1}, i_{3}, i_{4}, i_{5}\right\}\right) \Rightarrow i_{1} \in E_{i_{2}}^{\mu} \\
& \left\{i_{1}, i_{2}\right\} \in \mathcal{A}_{y}\left(\left\{i_{1}, i_{2}, i_{4}\right\}\right) \Rightarrow i_{2} \in E_{i_{3}}^{\mu} \\
& \left\{i_{2}, i_{3}\right\} \in \mathcal{A}_{x}\left(\left\{i_{1}, i_{2}, i_{3}, i_{5}\right\}\right) \Rightarrow i_{3} \in E_{i_{4}}^{\mu} \\
& \left\{i_{3}, i_{4}\right\} \in \mathcal{A}_{y}\left(\left\{i_{2}, i_{3}, i_{4}\right\}\right) \Rightarrow i_{4} \in E_{i_{1}}^{\mu} .
\end{aligned}
$$

Carrying out the above cycle yields the assignment

$$
\nu=\left(\begin{array}{ccccc}
i_{1} & i_{2} & i_{3} & i_{4} & i_{5} \\
x & y & x & y & i_{5}
\end{array}\right)
$$

which clearly Pareto dominates $\mu$. However, $\nu$ is not stable, because $\mathcal{A}_{x}\left(\left\{i_{1}, i_{3}, i_{5}\right\}\right)=$ $\left\{\left\{i_{1}, i_{5}\right\},\left\{i_{3}, i_{5}\right\}\right\}$, so $\left(x, i_{5}\right)$ blocks $\nu$. In fact, $\mu$ is constrained efficient. Note that the only Pareto improvements over $\mu$ are the following:

$$
\mu_{1}=\left(\begin{array}{ccccc}
i_{1} & i_{2} & i_{3} & i_{4} & i_{5} \\
x & y & y & x & i_{5}
\end{array}\right)
$$

$\mathcal{A}_{y}\left(\left\{i_{2}, i_{3}, i_{4}\right\}\right)=\left\{\left\{i_{2}, i_{4}\right\},\left\{i_{3}, i_{4}\right\}\right\}$, so $\left(b, i_{4}\right)$ blocks $\mu_{1}$.

$$
\mu_{2}=\left(\begin{array}{ccccc}
i_{1} & i_{2} & i_{3} & i_{4} & i_{5} \\
x & x & y & y & i_{5}
\end{array}\right)
$$

$\mathcal{A}_{x}\left(\left\{i_{1}, i_{2}, i_{3}\right\}\right)=\left\{\left\{i_{2}, i_{3}\right\}\right\}$, so $\left(x, i_{3}\right)$ blocks $\mu_{2}$.

$$
\mu_{3}=\left(\begin{array}{ccccc}
i_{1} & i_{2} & i_{3} & i_{4} & i_{5} \\
y & y & x & x & i_{5}
\end{array}\right)
$$


$\mathcal{A}_{x}\left(\left\{i_{1}, i_{3}, i_{4}\right\}\right)=\left\{\left\{i_{1}, i_{4}\right\}\right\}$, so $\left(x, i_{1}\right)$ blocks $\mu_{3}$.

$$
\mu_{4}=\left(\begin{array}{ccccc}
i_{1} & i_{2} & i_{3} & i_{4} & i_{5} \\
y & x & x & y & i_{5}
\end{array}\right)
$$

$\mathcal{A}_{y}\left(\left\{i_{1}, i_{2}, i_{4}\right\}\right)=\left\{\left\{i_{1}, i_{2}\right\},\left\{i_{2}, i_{4}\right\}\right\}$, so $\left(y, i_{2}\right)$ blocks $\mu_{4}$.

$$
\nu=\left(\begin{array}{ccccc}
i_{1} & i_{2} & i_{3} & i_{4} & i_{5} \\
x & y & x & y & i_{5}
\end{array}\right)
$$

$\nu$ is not stable as pointed out before. Hence, no stable assignment Pareto dominates $\mu$, so $\mu$ is constrained efficient.

Remark 6 When priorities are allowed to be substitutable, the stable improvement cycles as defined in Erdil and Ergin (2008) do not capture every stability preserving Pareto improvement.

Proof. Let $x, y, z, w$ be distinct schools with $q_{x}=q_{y}=2$ and $q_{z}=q_{w}=1$. We have six students: $i, j, k_{i}, k_{j}, \ell_{i}, \ell_{j}$ and an assignment $\mu$ :

$$
\mu=\left(\begin{array}{cccccc}
i & j & k_{i} & k_{j} & \ell_{i} & \ell_{j} \\
x & x & y & y & w & z
\end{array}\right)
$$

The priority structure is such that

$$
\mathcal{A}_{x}\left(\left\{\ell_{i}, \ell_{j}, i, j\right\}\right)=\{\{i, j\}\} \quad \mathcal{A}_{x}\left(\left\{\ell_{i}, \ell_{j}, j\right\}\right)=\left\{\left\{\ell_{i}, j\right\}\right\} \quad \mathcal{A}_{x}\left(\left\{\ell_{i}, \ell_{j}, i\right\}\right)=\left\{\left\{\ell_{j}, i\right\}\right\}
$$

and

$$
\mathcal{A}_{y}\left(\left\{k_{i}, k_{j}, i, j\right\}\right)=\left\{\left\{k_{i}, k_{j}\right\}\right\} \quad \mathcal{A}_{y}\left(\left\{i, j, k_{i}\right\}\right)=\left\{\left\{i, k_{i}\right\}\right\} \quad \mathcal{A}_{y}\left(\left\{i, j, k_{j}\right\}\right)=\left\{\left\{j, k_{j}\right\}\right\}
$$

Suppose the preferences $R$ are as below, where the boxes indicate the respective students' assignments under $\mu$ :

\begin{tabular}{c|c|c|c|c|c}
$R_{i}$ & $R_{j}$ & $R_{k_{i}}$ & $R_{k_{j}}$ & $R_{\ell_{i}}$ & $R_{\ell_{j}}$ \\
\hline$y$ & $y$ & $w$ & $z$ & $x$ & $x$ \\
$x$ & $x$ & $y$ & $y$ & $w$ & $z$
\end{tabular}

With these preferences, $\mu$ is stable with respect to $\mathcal{A}$, but obviously, letting each student get their most preferred school preserves stability, while Pareto improving over $\mu$. Can 

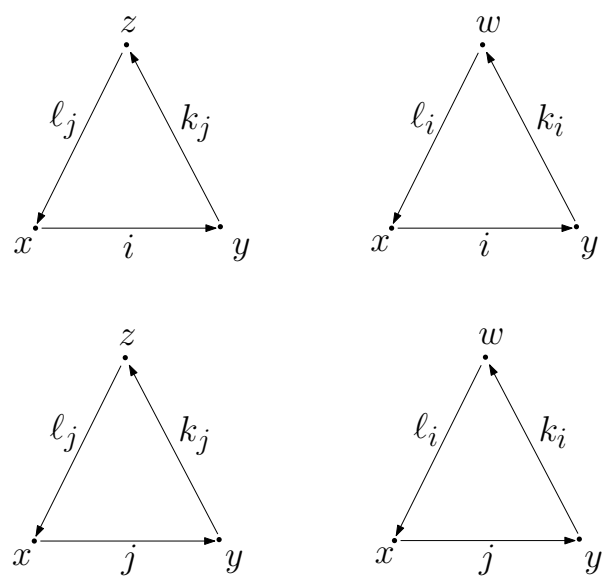

Figure 2: These cycles of schools are the only candidates for stable improvement cycles in the sense of Erdil and Ergin (2008).

this improvement be achieved through Erdil and Ergin's stable improvement cycles? The only "candidates" for such cycles are shown in Figure 2.

However, none of these cycles preserves stability. The first cycle fails to preserve stability, because $\mathcal{A}_{x}\left(\left\{\ell_{i}, \ell_{j}, j\right\}\right)=\left\{\left\{\ell_{i}, j\right\}\right\}$, and thus $\ell_{j}$ cannot replace $i$ at school $x$. The second cycle also fails to preserve stability, because $\mathcal{A}_{y}\left(\left\{i, j, k_{j}\right\}\right)=\left\{\left\{j, k_{j}\right\}\right\}$, and therefore $i$ cannot replace $k_{i}$. Likewise, the third one fails, because $j$ cannot replace $k_{j}$ at $y$, while for the fourth one $\ell_{i}$ cannot replace $j$ at $x$.

On the other hand, if all of the students were to move to their favorite schools at the same time, the resulting assignment would be stable. We can express this stability preserving move as the following cycle of students (as opposed to a cycle of schools ${ }^{21}$ as in Erdil and Ergin, 2008):

$$
i \rightarrow k_{j} \rightarrow \ell_{j} \rightarrow j \rightarrow k_{i} \rightarrow \ell_{i} \rightarrow i
$$

which features schools $x$ and $y$ twice each as opposed to a stable improvement cycle (as in Erdil and Ergin, 2008) which insists on schools being distinct ${ }^{22}$

Remark 7 Let $\mathcal{A}$ be an acceptant, substitutable and consistent priority structure. For all preference profiles, all constrained efficient assignments are efficient if and only if

\footnotetext{
${ }^{21}$ Note that this example does not rely on ties.

${ }^{22}$ Note that this example does not rely on ties.
} 
$\mathcal{A}$ does not have any generalized weak cycle, where we define a generalized weak cycle as a sequence of distinct schools $x_{0}, x_{1}, \ldots, x_{n-1} \in X$, and distinct students $j, i_{0}, i_{1}, \ldots, i_{n-1} \in N$ with $n \geq 2$ such that

(1) $x_{\ell} \neq x_{\ell+1}$ for $\ell \in\{0,1, \ldots, n-1\}\left(\right.$ with $\left.x_{n}=x_{0}\right)$,

(2) there exist mutually disjoint sets of students $S_{x_{0}}, \ldots, S_{x_{n-1}} \subseteq N \backslash\left\{j, i_{0}, i_{1}, \ldots, i_{n-1}\right\}$ such that

$$
\begin{aligned}
& j \notin \mathcal{C} \mathcal{A}_{x_{0}}\left(S_{x_{0}} \cup\left\{i_{0}, j\right\}\right) \\
& j \in \mathcal{C A}_{x_{0}}\left(S_{x_{0}} \cup\left\{i_{n-1}, j\right\}\right) \\
& i_{n-1} \notin \mathcal{C A}_{x_{0}}\left(S_{x_{0}} \cup\left\{i_{0}, i_{n-1}\right\}\right) \\
& \text { (C) } \quad i_{n-2} \notin \mathcal{C A}_{x_{n-1}}\left(S_{x_{n-1}} \cup\left\{i_{n-1}, i_{n-2}\right\}\right) \\
& \vdots \\
& \\
& i_{1} \notin \mathcal{C A}_{x_{2}}\left(S_{x_{2}} \cup\left\{i_{2}, i_{1}\right\}\right) \\
& i_{0} \notin \mathcal{C A}_{x_{1}}\left(S_{x_{1}} \cup\left\{i_{1}, i_{0}\right\}\right) \\
& \\
& \text { (S) } \quad\left|S_{x_{\ell}}\right|=q_{x_{\ell}}-1 \text { for } \ell=0,1, \ldots, n-1,
\end{aligned}
$$

where $\mathcal{C} \mathcal{A}_{x}(S)=\left\{i \in S \mid i \in S^{\prime}\right.$ for all $\left.S^{\prime} \in \mathcal{A}_{x}(S)\right\}$.

Proof. $(\Leftarrow)$ : Suppose, for a contradiction, that $\mu$ is constrained efficient, yet not Pareto efficient. Of all the Pareto improvements over $\mu$, let $\nu$ be one which has the least number of students improving over $\mu$. Denote by $N^{\prime}$ the set of students who are better off under $\nu$ compared with $\mu$ :

$$
N^{\prime}=\left\{i \mid \nu(i) P_{i} \mu(i)\right\}
$$

Denote by $\mathcal{E}_{j}^{\mu}$ the set of students who envy the student $j$ under $\mu$ :

$$
\mathcal{E}_{j}^{\mu}=\left\{\ell \in N \mid \mu(j) P_{\ell} \mu(\ell)\right\} .
$$

Set $\mathcal{E}_{j}^{\prime}$ to be the set of students in $N^{\prime}$ who envy $j$. That is,

$$
\mathcal{E}_{j}^{\prime}=\mathcal{E}_{j}^{\mu} \cap N^{\prime}=\left\{\ell \in N^{\prime} \mid \mu(j) P_{\ell} \mu(\ell)\right\} .
$$

Since $\mathcal{A}$ is acceptant, $\mu$ must be non-wasteful, and therefore the reshuffling lemm $2^{23}$ applies, i.e., any Pareto improvement over $\mu$ is due to reshuffling of already assigned seats

\footnotetext{
${ }^{23}$ See Erdil (2014).
} 
between their recipients. Therefore if $j \in N^{\prime}$, that is, if $j$ is part of an improvement, she must receive someone else's seat, whereas her seat must be reassigned to another person who necessarily is also better off. In other words we have $\mu(j) \in \nu\left(N^{\prime}\right)$. In particular, $\mu(j)$ is desired by some student in $N^{\prime}$ under $\mu$, and hence $\mathcal{E}_{j}^{\prime}$ is nonempty. Because $\mu$ is stable, we have

$$
\mu^{-1}(\mu(j)) \in \mathcal{A}_{\mu(j)}\left(\mathcal{E}_{j}^{\mu} \cup \mu^{-1}(\mu(j))\right) .
$$

Furthermore, $\mathcal{E}_{j}^{\prime} \subseteq \mathcal{E}_{j}^{\mu}$ and $\mathcal{A}$ being substitutable imply that

$$
\mu^{-1}(\mu(j)) \in \mathcal{A}_{\mu(j)}\left(\mathcal{E}_{j}^{\prime} \cup \mu^{-1}(\mu(j))\right) .
$$

Removing $j$ from the choice set, we conclude, again using substitutability, that $\mu^{-1}(\mu(j)) \backslash\{j\}$ is a subset of a chosen element from $\mathcal{E}_{j}^{\prime} \cup\left[\mu^{-1}(\mu(j)) \backslash\{j\}\right]$. In other words

$$
\mu^{-1}(\mu(j)) \backslash\{j\} \subseteq S^{\prime} \quad \text { for some } \quad S^{\prime} \in \mathcal{A}_{\mu(j)}\left(\mathcal{E}_{j}^{\prime} \cup\left[\mu^{-1}(\mu(j)) \backslash\{j\}\right]\right)
$$

Any such $S^{\prime}$ has exactly one element from $\mathcal{E}_{j}^{\prime}$, and let $E_{j}^{\prime}$ be the set of those elements:

$$
E_{j}^{\prime}=\left\{\begin{array}{c|c}
\ell \in \mathcal{E}_{j}^{\prime}, \quad \text { and }\left[\mu^{-1}(\mu(j)) \backslash\{j\}\right] \cup\{\ell\}=S^{\prime} \\
\text { for some } S^{\prime} \in \mathcal{A}_{\mu(j)}\left(\mathcal{E}_{j}^{\prime} \cup\left[\mu^{-1}(\mu(j)) \backslash\{j\}\right]\right)
\end{array}\right\}
$$

Thus, $E_{j}^{\prime}$ is a nonempty subset of $N^{\prime}$ for each $j \in N^{\prime}$. Consider a directed graph whose set of vertices is $N^{\prime}$. For each $i \in E_{j}^{\prime}$, let there be a directed edge from $i$ to $j$. Therefore, every vertex in this graph has an incoming edge, and since it is a finite graph, there must be a cycle. Since $\nu$ is Pareto improvement over $\mu$ which has the least number of students improving, all students in $N^{\prime}$ is in a single cycle of the above graph. Suppose this cycle consists of students $i_{0}, i_{1}, \ldots, i_{n-1}, i_{n}=i_{0}$, where $n \geq 2$, and there is an edge from $i_{\ell}$ to $i_{\ell+1}$ for $\ell=0,1, \ldots, n-1$. Denoting $\mu\left(i_{\ell}\right)=x_{\ell}$, since $i_{\ell}$ envies $i_{\ell+1}$, we have $x_{\ell} \neq x_{\ell+1}$ for each $\ell$. In fact, these schools $x_{0}, \ldots, x_{n-1}$ must be distinct, for otherwise we would have a shorter cycle, which would give a Pareto improvement over $\mu$, involving a smaller number of students improving. To be more precise, if $x_{0}=x_{k}$ for some $k \leq n-1$, then the cyclic trade which allows $i_{\ell}$ take $x_{\ell+1}$ for $\ell=0, \ldots, k-1$, and letting $i_{k}$ take $x_{0}$ would lead to a Pareto improvement over $\mu$. Since $k<n$, this would contradict with the assumption that $\nu$ was the "smallest" improvement over $\mu$. Since $\mu\left(i_{\ell}\right)=x_{\ell}$, the students $i_{0}, \ldots, i_{n-1}$ are necessarily distinct. 
The fact that $\mu$ is stable with respect to acceptant priorities implies that it is non-wasteful. Since each $x_{\ell}$ is desired by some student at assignment $\mu$, all seats at these schools must be assigned under $\mu$. Denoting $S_{x_{\ell}}=\mu^{-1}\left(x_{\ell}\right) \backslash\left\{i_{\ell}\right\}$, we know that $S_{x_{0}}, \ldots, S_{x_{n-1}}$ are mutually disjoint subsets of $N \backslash\left\{i_{0}, i_{1}, \ldots, i_{n-1}\right\}$, because $x_{0}, x_{1}, \ldots, x_{n-1}$ are distinct schools. Moreover we have

$$
\begin{aligned}
& \text { (1) }\left|S_{x_{\ell}}\right|=q_{x_{\ell}}-1, \\
& \text { (2) } i_{n-1} \notin \mathcal{C A}_{x_{0}}\left(S_{x_{0}} \cup\left\{i_{0}, i_{n-1}\right\}\right) \\
& i_{n-2} \notin \mathcal{C A}_{x_{n-1}}\left(S_{x_{n-1}} \cup\left\{i_{n-1}, i_{n-2}\right\}\right) \\
& \vdots \\
& i_{1} \notin \mathcal{C A}_{x_{2}}\left(S_{x_{2}} \cup\left\{i_{2}, i_{1}\right\}\right) \\
& i_{0} \notin \mathcal{C A}_{x_{1}}\left(S_{x_{1}} \cup\left\{i_{1}, i_{0}\right\}\right)
\end{aligned}
$$

because otherwise, if student $i_{\ell}$ were to be in $\mathcal{C A}_{x_{\ell+1}}\left(S_{x_{\ell+1}} \cup\left\{i_{\ell+1}, i_{\ell}\right\}\right)$ for some $\ell$, then we would have $S_{x_{\ell+1}} \cup\left\{i_{\ell+1}\right\} \notin \mathcal{A}_{x_{\ell+1}}\left(S_{x_{\ell+1}} \cup\left\{i_{\ell+1}, i_{\ell}\right\}\right)$, contradicting the fact that $\mu$ is stable.

Let $\omega$ be the assignment derived from $\mu$ by letting the students $i_{0}, i_{1}, \ldots, i_{n-1}$ exchange their schools along the improvement cycle suggested above. In other words,

$$
\omega(i)= \begin{cases}\mu(i) & i \neq i_{\ell} \\ \mu\left(i_{\ell+1}\right) & i=i_{\ell}\end{cases}
$$

$\omega$ Pareto dominates $\mu$, whereas $\mu$ is constrained efficient, so $\omega$ is not stable. Therefore the cyclic trade letting $i_{\ell}$ take $\mu\left(i_{\ell+1}\right)$ for $\ell=0,1, \ldots, n-1, n \equiv 0$ cannot be preserving stability. All school priorities are acceptant, and the new assignment $\omega$ is clearly individually rational. Therefore we know from Proposition 1 that there must be a blocking pair involving one of these schools. Suppose that $j$ and $x_{0}$ form a blocking pair for $\omega$, so $\omega^{-1}\left(x_{0}\right) \notin \mathcal{A}_{x}\left(\omega^{-1}\left(x_{0}\right) \cup\{j\}\right)$. Then $x_{0} P_{j} \omega(j)$ and

$$
j \in \mathcal{C A}_{x_{0}}\left(\omega^{-1}\left(x_{0}\right) \cup\{j\}\right)=\mathcal{C A}_{x_{0}}\left(S_{x_{0}} \cup\left\{i_{n-1}, j\right\}\right) .
$$

First, note that $j \neq i_{n-1}$, because $\omega\left(i_{n-1}\right)=x_{0} P_{j} \omega(j)$. Secondly, $j \neq i_{0}$, because $\omega\left(i_{0}\right) P_{i_{0}} \mu\left(i_{0}\right)=x_{0}$, while $x_{0} P_{j} \omega(j)$. And lastly if $j=i_{k}$ for some $k \in\{1, \ldots, n-2\}$, then we have an envy cycle

$$
i_{0} \rightarrow i_{1} \rightarrow \cdots \rightarrow i_{k} \rightarrow i_{0}
$$


which would allow a Pareto improvement involving only $k+1 \leq n-1$ students, contradicting our earlier choice of a smallest Pareto improvement over $\mu$. Thus $j \notin$ $\left\{i_{0}, \ldots, i_{n-1}\right\}$.

Furthermore, the fact that $\mu$ is stable implies

$$
j \notin \mathcal{C} \mathcal{A}_{x_{0}}\left(S_{x_{0}} \cup\left\{i_{0}, j\right\}\right)
$$

Thus, combining $(*),(* *)$, and $(* * *)$, we have a generalized weak cycle

$$
\begin{aligned}
& j \notin \mathcal{C} \mathcal{A}_{x_{0}}\left(S_{x_{0}} \cup\left\{i_{0}, j\right\}\right) \\
& j \in \mathcal{C} \mathcal{A}_{x_{0}}\left(S_{x_{0}} \cup\left\{i_{n-1}, j\right\}\right) \\
& i_{n-1} \notin \mathcal{C} \mathcal{A}_{x_{0}}\left(S_{x_{0}} \cup\left\{i_{0}, i_{n-1}\right\}\right) \\
& i_{n-2} \notin \mathcal{C} \mathcal{A}_{x_{n-1}}\left(S_{x_{n-1}} \cup\left\{i_{n-1}, i_{n-2}\right\}\right) \\
& \quad \vdots \\
& i_{1} \notin \mathcal{C A}_{x_{2}}\left(S_{x_{2}} \cup\left\{i_{2}, i_{1}\right\}\right) \\
& i_{0} \notin \mathcal{C} \mathcal{A}_{x_{1}}\left(S_{x_{1}} \cup\left\{i_{1}, i_{0}\right\}\right)
\end{aligned}
$$

with $\left|S_{x_{\ell}}\right|=q_{x_{\ell}}-1$ for $\ell=0,1, \ldots, n-1$.

$(\Rightarrow)$ : Let $N, X$, and $q$ and $\mathcal{A}$ be given. Assume that $\mathcal{A}$ has a generalized weak cycle, that is, there exist distinct schools $x_{0}, x_{1}, \ldots, x_{n-1} \in X$ and distinct students $j, i_{0}, i_{1}, \ldots, i_{n-1} \in N$ with $n \geq 2$ such that

(1) $x_{\ell} \neq x_{\ell+1}$ for $\ell \in\{0,1, \ldots, n-1\}\left(\right.$ with $\left.x_{n}=x_{0}\right)$,

(2) there exist mutually disjoint sets of students $S_{x_{0}}, \ldots, S_{x_{n-1}} \subseteq N \backslash\left\{j, i_{0}, i_{1}, \ldots, i_{n-1}\right\}$ such that

$$
\begin{aligned}
& j \notin \mathcal{C} \mathcal{A}_{x_{0}}\left(S_{x_{0}} \cup\left\{i_{0}, j\right\}\right) \\
& j \in \mathcal{C A}_{x_{0}}\left(S_{x_{0}} \cup\left\{i_{n-1}, j\right\}\right) \\
& i_{n-1} \notin \mathcal{C A}_{x_{0}}\left(S_{x_{0}} \cup\left\{i_{0}, i_{n-1}\right\}\right) \\
& (\mathbf{C}) \quad i_{n-2} \notin \mathcal{C} \mathcal{A}_{x_{n-1}}\left(S_{x_{n-1}} \cup\left\{i_{n-1}, i_{n-2}\right\}\right) \\
& \quad \vdots \\
& i_{1} \notin \mathcal{C} \mathcal{A}_{x_{2}}\left(S_{x_{2}} \cup\left\{i_{2}, i_{1}\right\}\right) \\
& i_{0} \notin \mathcal{C} \mathcal{A}_{x_{1}}\left(S_{x_{1}} \cup\left\{i_{1}, i_{0}\right\}\right) \\
& \quad \\
& \text { (S) } \quad\left|S_{x_{\ell}}\right|=q_{x_{\ell}}-1 \text { for } \ell=0,1, \ldots, n-1 .
\end{aligned}
$$


Consider the preference profile $R$ where students in $S_{x_{0}}, \cdots, S_{x_{n-1}}$, respectively, rank $x_{0}, \cdots, x_{n-1}$ as their top choice, and the preferences of $j, i_{0}, i_{1}, \cdots, i_{n-1}$ are such that $x_{0} P_{j} j P_{j} \cdots$, and $x_{\ell+1} P_{i_{\ell}} x_{\ell} P_{i_{\ell}} i_{\ell} P_{i_{\ell}} \cdots$, for $\ell=0,1, \ldots, n-1$. Finally, let students outside a generalized weak cycle prefer not to be assigned to any school. Consider the assignment $\mu$ such that $\mu(j)=j$ and for each $k_{\ell} \in S_{x_{\ell}} \cup\left\{i_{\ell}\right\}$ one has $\mu\left(k_{\ell}\right)=x_{\ell}$ for $\ell=0,1, \ldots, n-1$. Now the only candidates for blocking pairs are $\left(j, x_{0}\right)$ and $\left(i_{\ell}, x_{\ell+1}\right)$ for $\ell=0,1, \ldots, n-1$. However, the generalized weak cycle conditions are such that $j \notin \mathcal{C} \mathcal{A}_{x_{0}}\left(S_{x_{0}} \cup\left\{i_{0}, j\right\}\right)$ and $i_{\ell} \notin \mathcal{C A}_{x_{\ell+1}}\left(S_{x_{\ell+1}} \cup\left\{i_{\ell+1}, i_{\ell}\right\}\right)$ for $\ell=0,1, \ldots, n-1$, ensuring that $\mu$ is stable.

Moreover, there is only one assignment that Pareto dominates $\mu$, namely the assignment $\nu$ obtained from $\mu$ by letting $i_{\ell}$ be assigned to $\mu\left(i_{\ell+1}\right)$ for $\ell=0,1, \ldots, n-1$. Since $j \in \mathcal{C A}_{x_{0}}\left(S_{x_{0}} \cup\left\{i_{n-1}, j\right\}\right), x_{0} P_{j} \nu(j)=\mu(j)=j$ and $\nu^{-1}\left(x_{0}\right)=S_{x_{0}} \cup\left\{i_{n-1}\right\}$, the assignment $\nu$ is not stable. Thus $\mu$ is constrained efficient, but not Pareto efficient. 